\author{
铱杂碳龙配合物的合成及反应性 \\ 李金华 $a$ 卓庆德 ${ }^{b}$ 卓凯玥 $b$ 陈大发 ${ }^{*}, a$ 夏海平 $*, a, b$ \\ ( ${ }^{a}$ 南方科技大学化学系 深圳格拉布斯研究院 深圳 518055) \\ ( ${ }^{b}$ 厦门大学化学系 厦门 361005)
}

\begin{abstract}
摘要 碳龙配合物由一条碳数不少于 7 的碳链配体鳌合过渡金属而成, 代表了一系列全新的 $\pi$ 共轭分子骨架基元, 并 表现出独特的光物理性质, 具有很好的应用前景. 通过合理设计多炔化合物, 实现了 “一锅法” 构筑具有大 $\pi$ 共轭体系 的铱杂碳龙配合物 4 和 5 , 首次将碳龙配合物拓展至金属铱体系. 此外, 还研究了 4 和 $\mathbf{5}$ 的配体取代反应, 制备了铱杂 碳龙配合物 6 9. 所制备的一系列铱杂碳龙配合物在紫外-可见区具有很好的吸收, 部分甚至在近红外区也表现出一定 的吸收特性. 光热性能研究表明, 化合物 $\mathbf{5}$ 具有较好的近红外光热效果: 在 $808 \mathrm{~nm}$ 的激光照射下, 含有 $\mathbf{5}\left(0.1 \mathrm{mg} \cdot \mathrm{mL}^{-1}\right)$ 的乙醇/水溶液在 $10 \mathrm{~min}$ 内温度可升高近 $40{ }^{\circ} \mathrm{C}$, 这为后续铱杂碳龙配合物的应用研究提供了可能性.
\end{abstract}

关键词 碳龙配合物; 一锅法合成; 铱; 多炔化合物; 光热性能

\title{
Synthesis and Reactivity Studies of Irida-carbolong Complexes
}
Li, Jinhua ${ }^{a}$
Zhuo, Qingde ${ }^{b}$
Zhuo, Kaiyue ${ }^{b}$
Chen, Dafa*,a
Xia, Haiping*,a,b

( ${ }^{a}$ Department of Chemistry, Shenzhen Grubbs Institute, Southern University of Science and Technology, Shenzhen 518055)

( ${ }^{b}$ Department of Chemistry, College of Chemistry and Chemical Engineering, Xiamen University, Xiamen 361005)

\begin{abstract}
The term "carbolong complexes" represents a series of novel $\pi$-conjugating aromatic frameworks, which are formed by the chelation of a carbon chain (carbolong ligand) containing not less than 7 carbon atoms with a transition metal fragment. Carbolong complexes show unique photophysical properties, thus exhibiting promising potential applications. Inspired by the efficient strategies for the construction of Os-, Ru-, and Rh-carbolong complexes by the reactions of multiynes with simple metal sources, we describe here the reasonable design of two multiyne compounds $\mathbf{1}$ and $\mathbf{2}$, which could react with the iridium precursor $\left[\operatorname{Ir}\left(\mathrm{CH}_{3} \mathrm{CN}\right)(\mathrm{CO})\left(\mathrm{PPh}_{3}\right)_{2}\right] \mathrm{BF}_{4}(\mathbf{3})$ to produce the irida-carbolong complexes 4 and 5 with a large $\pi$ conjugation system via a "one-pot" method, respectively. This is the first time to expand the carbolong complex to iridium. In addition, the ligand substitution reactions of $\mathbf{4}$ and $\mathbf{5}$ have been investigated. Treatment of corresponding ligands with $\mathbf{4}$ and $\mathbf{5}$ resulted in the generation of several new irida-carbolong complexes $\mathbf{6} \sim \mathbf{9}$. All the prepared irida-carbolong complexes were fully characterized by NMR and HRMS. The molecular structures of the irida-carbolong complexes $\mathbf{4} \sim \mathbf{8}$ were further confirmed by single crystal X-ray diffractions. A possible mechanism was proposed for the formation of the irida-carbolong complexes on the basis of the X-ray characterization of the key intermediate Int 2. Due to the unique large $\pi$ conjugation system, all the synthesized irida-carbolong complexes exhibit remarkable absorption properties in the ultraviolet visible region, and some of them even show considerable absorption characteristics in the near infrared region (NIR). The consequently photothermal property investigation indicates that complex $\mathbf{5}$ has a good NIR photothermal effect: by measuring the temperature of its solution under the NIR laser irradiation $\left(808 \mathrm{~nm}, 1.0 \mathrm{~W} \cdot \mathrm{cm}^{-2}\right)$, the temperature of ethanol/water solution containing $5\left(0.1 \mathrm{mg} \cdot \mathrm{mL}^{-1}\right)$ can be increased by nearly $40{ }^{\circ} \mathrm{C}$ in $10 \mathrm{~min}$, providing numerous possibilities for the follow-up application research based on carbolong complexes.
\end{abstract}

Keywords carbolong complex; "one-pot" method; iridium; multiyne; photothermal property

\section{1 引言}

金属杂芳香化合物因兼具芳香性和金属有机反应 性的特点, 吸引了越来越多学者的关注. 早期对于金属 杂芳香化合物的研究主要局限于金属苯 ${ }^{[1]}$ 、金属苯炔 ${ }^{[2]}$ 、 金属荎 ${ }^{[3]}$ 以及含有主族杂原子的金属杂芳环 ${ }^{[4]}$. 随着研 究的不断深入, 一些具有新颖结构的金属杂芳香化合物
应运而生，如金属杂环戊二烯双负离子 ${ }^{[5]}$ 、金属杂螺环 化合物 ${ }^{[6]}$ 、双金属杂轮烯 ${ }^{[7]}$ 、金属杂环丙烯 ${ }^{[8]}$ 和金属杂稠 环芳烃 ${ }^{[9]}$ 等, 这极大地丰富了金属杂芳香化学.

近年来，一系列具有全新芳香骨架基元的金属杂芳 香化合物——碳龙配合物(carbolong complexes)相继被 报道 ${ }^{[10]}$. 碳龙配合物的定义为: 由一条碳数不少于 7 的

*E-mail: chendf@sustech.edu.cn; xiahp@sustech.edu.cn.

Received August 26, 2020; published September 24, 2020.

Supporting information for this article is available free of charge via the Internet at http://sioc-journal.cn.

Project supported by the National Natural Science Foundation of China (Nos. U1705254, 21931002, 21871068), the Guangdong Provincial Key Laboratory of Catalysis (No. 2020B121201002).

国家自然科学基金(Nos.: U1705254, 21931002, 21871068), 广东省催化化学重点实验室(No. 2020B121201002)资助项目. 
共轭碳链(碳龙配体)通过至少三个金属一碳键螯合过渡 金属形成的配合物，其特点是过渡金属的 $\mathrm{d}$ 轨道参与了 $\pi$ 共轭. 首例被报道的碳龙配合物为锇杂戊搭炔, 因其 碳龙配体为七碳链, 故也称为 7 碳龙配合物 ${ }^{[11]}$. 经过多 年的发展, $8 \sim 12$ 碳龙配合物也相继被合成(Figure 1). 碳龙配合物具有独特的光物理性质, 并已成功应用于光 声成像 ${ }^{[12]}$ 、光热治疗 ${ }^{[13]}$ 、单分子电子学 ${ }^{[14]}$ 及高分子自修 复材料 ${ }^{[15]}$ 等多个领域.

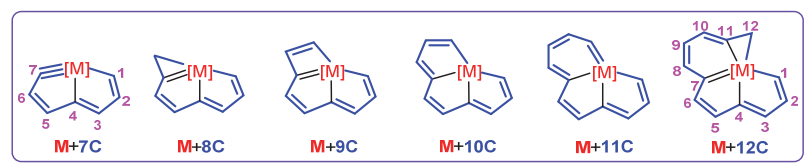

图 1 7 12 碳龙配合物示意图

Figure 1 The sketch map of $7 \mathrm{C} \sim 12 \mathrm{C}$ carbolong complexes

2017 年, 我们发展了由多炔碳链直接鳌合过渡金 属合成碳龙配合物的新策略, 这一策略实现了温和条件 下 “一锅法” 高效构筑不超过三个并环的锇杂碳龙配合 物 ${ }^{[16]}$. 不仅如此, 该方法还可用于钉杂碳龙配合物 ${ }^{[17]}$ 和 铑杂碳龙配合物 ${ }^{[18]}$ 的合成, 这拓展了碳龙化学的研究 空间. 为了进一步丰富碳龙化学, 本文设计合成了两个 新颖的多炔有机物, 通过它们与 $\left[\mathrm{Ir}\left(\mathrm{CH}_{3} \mathrm{CN}\right)(\mathrm{CO})\right.$ $\left.\left(\mathrm{PPh}_{3}\right)_{2}\right] \mathrm{BF}_{4}$ 的反应, “一锅法”合成了具有四个共轭并环 结构的铱杂碳龙配合物, 并研究了它们的反应性及光热 性能. 这些碳龙配合物具有刚性骨架及易修饰等特性, 在紫外-可见区甚至近红外区表现出较好的吸收特性, 并展现出良好的光热转化效果, 在光电转换材料领域具 有很好的应用前景. 这不仅是首次实现含四个共轭并环 的碳龙配合物 “一锅法” 合成, 也是首次将碳龙化学拓 展至金属 Ir 体系.

\section{2 结果与讨论}

\section{1 多炔化合物 1 和 2 的合成}

共轭程度的增加通常能够提升化合物的稳定性, 同 时还能改善其光物理性质. 为了合成具有更多共轭并环 的碳龙配合物, 我们将苯环引入到碳龙前体中, 合成了 如 Scheme 1 所示的两种多炔化合物 1 和 2, 详细合成步 骤以及表征见实验部分及支持信息 Figures $\mathrm{S} 1 \sim \mathrm{S} 18$.

\section{2 铱杂碳龙配合物 4 和 5 的合成}

成功合成多炔化合物 1 和 2 后，我们进一步探究了 它们与铱金属源 $\left[\operatorname{Ir}\left(\mathrm{CH}_{3} \mathrm{CN}\right)(\mathrm{CO})\left(\mathrm{PPh}_{3}\right)_{2}\right] \mathrm{BF}_{4}(3)$ 的反应 性. 如 Scheme 2 所示, 在常温下往 3 和三苯基膦的二氯 甲烷溶液中加入多炔化合物 1 , 溶液颜色很快由黄色变 成红色. 继续反应 $30 \mathrm{~min}$ 后加入过量氟硼酸水溶液, 反 应体系立即由红色变成绿色. 反应结束后经浓缩、乙醚 洗涤和真空干燥后得到绿色固体 4, 产率为 $85 \%$. 化合 物 4 的结构得到了核磁、X-射线单晶衍射结构以及高分 辨质谱表征。

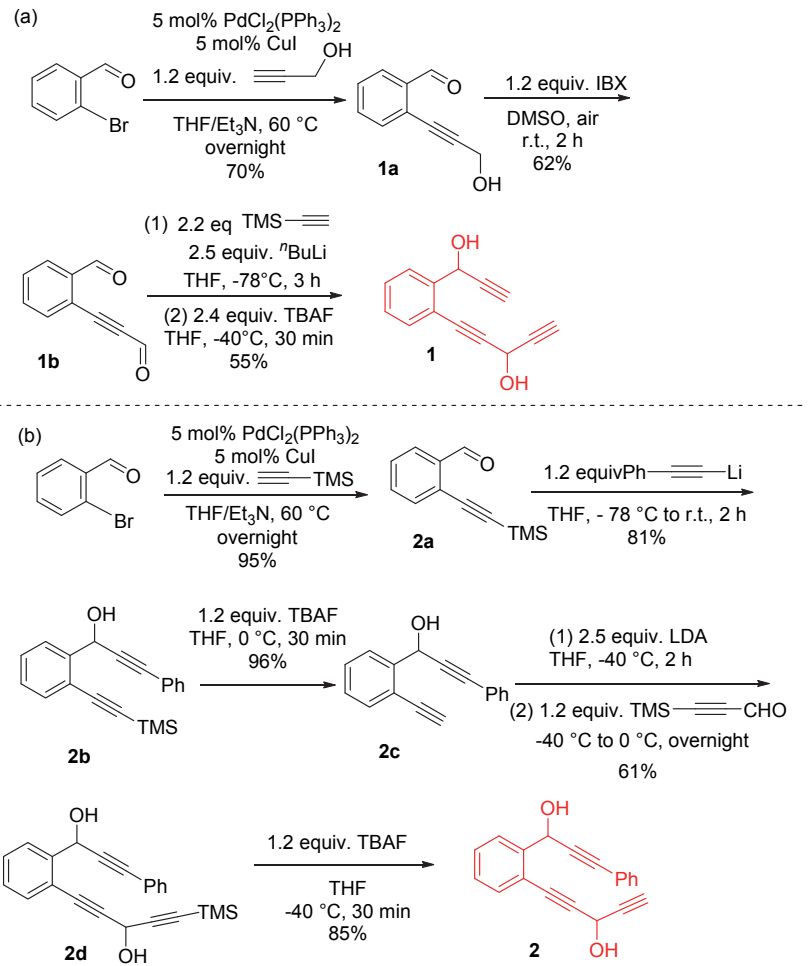

图式 1 多炔化合物 1 (a) 和 2 (b)的合成

Scheme 1 Synthesis of multiyne compounds 1 (a) and 2 (b)

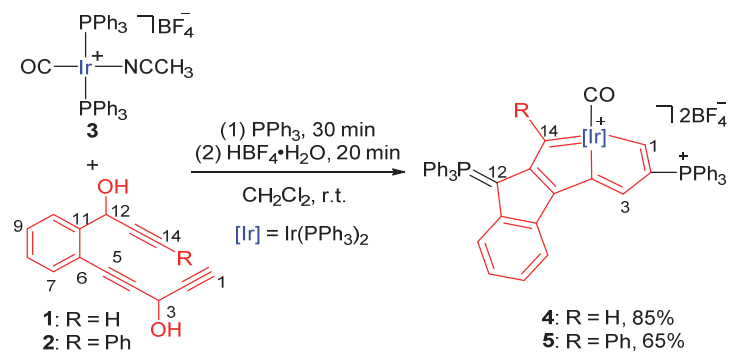

图式 2 铱杂碳龙配合物 4 和 5 的合成

Scheme 2 Synthesis of irida-carbolong complexes $\mathbf{4}$ and $\mathbf{5}$

在配合物 4 的 ${ }^{1} \mathrm{H}$ NMR 谱图中，14-H、1-H 和 3-H 这三个氢化学位移分别位于 $10.62,9.80$ 和 8.42 处; 在 ${ }^{31} \mathrm{P}\left\{{ }^{1} \mathrm{H}\right\}$ NMR 中, 两个季鏻的信号分别位于 13.45 和 6.66, 而金属中心两个等价膦的信号则位于 1.98 ; 在 ${ }^{13} \mathrm{C}\left\{{ }^{1} \mathrm{H}\right\} \mathrm{NMR}$ 中, 金属杂双五元环内与金属直接相连的 三个碳原子的化学位移分别位于 177.5 (1-C), 170.3 (4-C)和 195.2 (14-C)处, 而其它四个碳原子的化学位移 则分别位于 121.8 (2-C), 139.4 (3-C), 158.2 (5-C)和 173.6 (13-C)处. 这些数据与铑杂戊搭烯的数据类似 ${ }^{[18]}$, 验证 了化合物 4 中金属杂双五元的共轭性. 此外, 12-C 的化 学位移为 73.5 , 受到季鏻耦合作用裂分为双峰, 耦合常 数为 $118.2 \mathrm{~Hz}$, 这与文献报道的具有类似结构的 9-芴基 膦中相应碳原子的化学位移接近 ${ }^{[19]}$.

配合物 4 的结构进一步得到了 X-射线单晶衍射的 确认. 如 Figure 2 所示, 其主环平面由四个并环组成, 
金属被两个五元环所共用. 由 $\mathrm{Ir} 1$ 和 $\mathrm{C}(1) \sim \mathrm{C}(14)$ 十五个 原子组成的主环平面具有很高的共平面性, 其偏离拟合 平面的均方根偏差仅为 $0.032 \AA$. 由键长数据可知(Table $\mathrm{S} 3)$, 主环内 $\mathrm{C}-\mathrm{C}$ 键的键长 $(1.347 \sim 1.459 \AA)$ 介于单键 和双键之间, 并位于具有离域结构的铱杂苯中 $\mathrm{C}-\mathrm{C}$ 键 的键长范围内 $(1.335 \sim 1.464 \AA)^{[20]}$, 因此它们表现出了 一定的离域性. 此外, 金属杂双五元环中的 $\operatorname{Ir}(1)-\mathrm{C}(1)$ (2.085(4) $\AA), \operatorname{Ir}(1)-\mathrm{C}(4)(2.077(4) \AA)$ 和 $\operatorname{Ir}(1)-\mathrm{C}(14)$ (2.081(4) $\AA$ )的键长接近, 尽管略长于具有离域结构的铱 杂苯中的最长 $\mathrm{Ir}=\mathrm{C}$ 双键 $(2.066(13) \AA)$ 键长 ${ }^{[21]}$, 但短于 已报道的两例非芳香铱杂稠双五元环中 $\mathrm{Ir}-\mathrm{C}\left(\mathrm{sp}^{2}\right)$ 单键 的键长 $(2.100 \AA \text { 和 } 2.105 \AA)^{[22]}$, 因此, 4 中的 $\mathrm{Ir}-\mathrm{C}$ 键也 具有一定的离域特性. 有趣的是, 多炔化合物 1 中与 C12 连接的羟基在反应过程中被三苯基膦所取代, 导致 配合物 4 中含有一个 $\mathrm{C}(12)-\mathrm{P}(4)$ 键, 且它的键长 (1.740(4) Å) 也介于单键和双键之间: 短于 $\mathrm{C}(2)-\mathrm{P}(3)$ 单 键键长(1.801(4) $\AA$ ), 但是长于具有类似结构的 9-芴基膦 中 $\mathrm{C}=\mathrm{P}$ 双键的键长 $(1.7160(19) \AA \text { 和 1.7237(15) } \AA)^{[19]}$. 高分辨质谱数据与它的单晶结构吻合(实验值 722.1885 , 二价阳离子的理论值 722.1880).

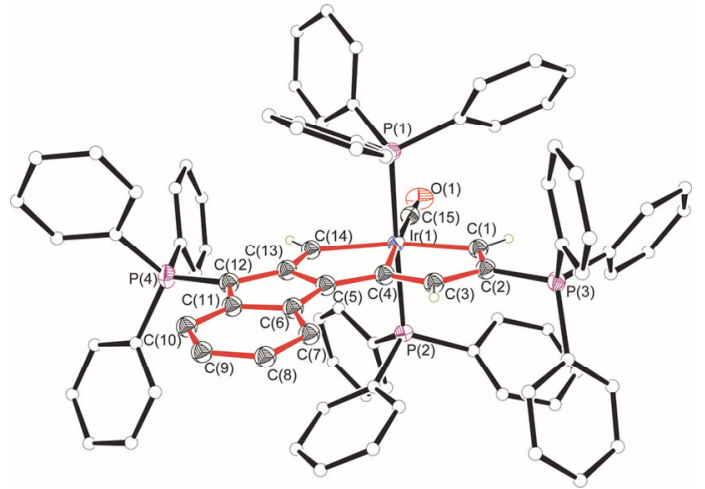

图 2 铱杂碳龙配合物 $\mathbf{4}$ 的阳离子晶体结构

Figure 2 Molecular structure for the cation of irida-carbolong complex 4. Ellipsoids at the $50 \%$ probability level. Counteranion and hydrogen atoms in $\mathrm{PPh}_{3}$ are omitted for clarity.

以上结果表明, 配合物 $\mathbf{4}$ 具有如 Scheme 3 所示的 $\mathbf{A}$ 和 $\mathbf{B}$ 两种共振式, 它可以看成是含有铱杂戊搭烯结构单 元且具有四个并环结构的大 $\pi$ 共轭体系. 这是首次通过 过渡金属源与多炔化合物经 “一锅法” 反应合成含四个 共轭并环的碳龙配合物, 也是首次将碳龙化学拓展至金 属 Ir 体系.

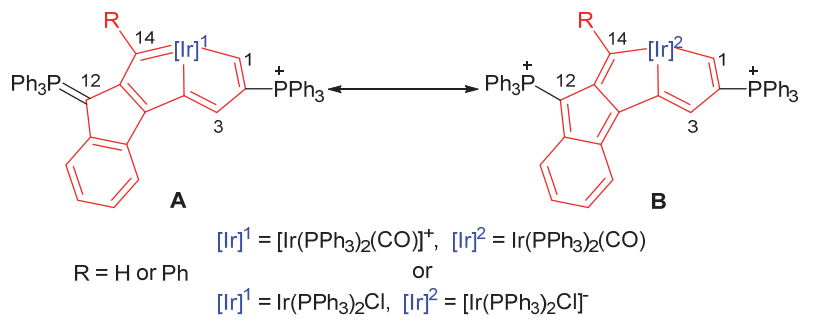

图式 3 铱杂碳龙配合物阳离子的共振式

Scheme 3 Resonance structures of the irida-carbolong cations.
类似地，铱杂碳龙配合物 $\mathbf{5}$ 也可以由铱金属源 $\mathbf{3}$ 与 多炔化合物 2 经“一锅法”合成，产率为 $65 \%$ (Scheme 2). 配合物 5 的结构与 4 类似, 它也具有 $\mathbf{A}$ 和 $\mathbf{B}$ 两种共振式 (Scheme 3). 需要指出的是, 与 4 相比, 配合物 $\mathbf{5}$ 中由 $\operatorname{Ir}(1)$ 和 $\mathrm{C}(1) \sim \mathrm{C}(14)$ 十五个原子组成的主结构共面性稍 差, 这可能是由于 $\mathrm{C}(14)$ 上苯基取代基的引入导致的, 其偏离拟合平面的均方根偏差为 $0.099 \AA$ (Figure 3). 此 外，尽管 5 中 $\mathrm{C} 14$ 上苯基的吸电子作用导致它的 $\operatorname{Ir}(1)$ - $\mathrm{C}(14)$ 键更倾向于单键化, 使该键的键长 $(2.135(7) \AA)$ 要长于 4 中 $\operatorname{Ir}(1)-\mathrm{C}(14)$ 键的键长(2.081(4) $\AA)$; 但是由 于苯基的吸电子作用同时也降低了 $\mathrm{C}(14)$ 原子的电子云 密度, 去屏蔽效应促使 5 中 14-C 的化学位移(212.3)却要 比 4 中 14-C 的化学位移(195.2)处于更低场.

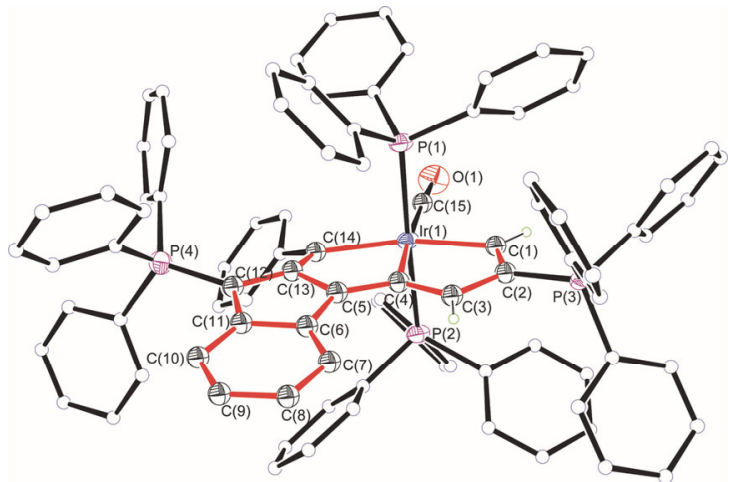

图 3 铱杂碳龙配合物 5 的阳离子晶体结构

Figure 3 Molecular structure for the cation of irida-carbolong complex $\mathbf{5}$. Ellipsoids at the $50 \%$ probability level. Counteranion and hydrogen atoms in $\mathrm{PPh}_{3}$ are omitted for clarity.

\section{3 铱杂碳龙配合物 4 和 5 的反应性}

成功制备了配合物 4 和 $\mathbf{5}$ 之后, 我们继续研究了它 们的反应性. 在脱羰基试剂二水合氧化三甲胺的作用 下, 4 和 5 均可以和四丁基氯化铵反应，分别生成产物 6 和 7, 分离收率分别为 $90 \%$ 和 $91 \%$ (Scheme 4). 在配合物 6 中, 金属中心两个等价膦的 ${ }^{31} \mathrm{P}$ 信号位于 -3.67 处, 这 与 4 相比具有较明显的变化, 因为它的金属中心配位环 境发生了改变. 有趣的是, 在 6 的 ${ }^{1} \mathrm{H} \mathrm{NMR}$ 和 ${ }^{13} \mathrm{C}\left\{{ }^{1} \mathrm{H}\right\}$ NMR 谱图中, 不论是 14-H 和 1-H 的化学位移(分别位于 12.94 和 11.23)还是 14-C 和 1-C 的化学位移(分别位于 225.1 和 197.3), 相较于 4 而言, 均移向了低场, 且与铱 杂苯中的卡宾碳的化学位移接近 ${ }^{[20]}$, 这表明配合物 6 中

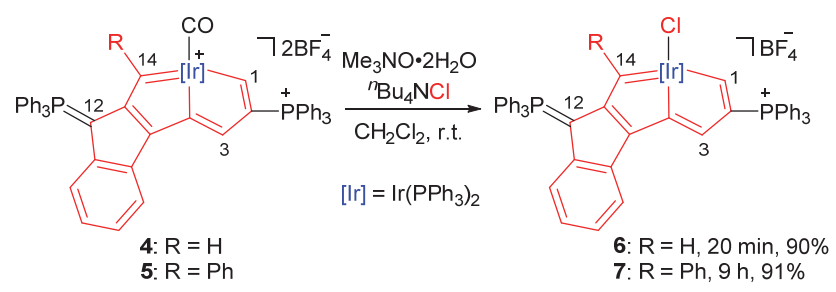

图式 4 铱杂碳龙配合物 6 和 7 的合成

Scheme 4 Synthesis of irida-carbolong complexes 6 and 7 
的 14-C 卡宾性质更加明显，即相对于 4 而言，6 更倾向 于以共振式 A 形式存在(Scheme 3).

配合物 6 的阳离子结构如 Figure 4 所示. 它的主结 构与 4 的阳离子结构类似，仅仅是金属中心的一个 $\mathrm{CO}$ 变成了氯配体. 主结构仍具有很好的平面性, 由 $\operatorname{Ir}(1)$ 和 $\mathrm{C}(1)-\mathrm{C}(14)$ 十五个原子组成的平面偏离拟合平面的均 方根偏差仅为 $0.049 \AA$. 与 4 相比, 配合物 6 中的 $\operatorname{Ir}(1)$ - C(1) (2.045(6) $\AA$ ) 和 $\operatorname{Ir}(1)-\mathrm{C}(14)(2.067(6) \AA)$ 的键长稍 短, 表明其双键性质更为明显, 这与它的 NMR 数据是 吻合的, 进一步验证了 $\mathbf{6}$ 更倾向于以共振式 $\mathbf{A}$ 形式存在 (由于配合物 7 具有与 6 类似的结构, 在这里我们不作详 细讨论, 它的单晶结构见 Figure S43). 值得一提的是, 铱杂碳龙配合物 $4 \sim 6$ 与锇杂 7 碳龙配合物 ${ }^{[23]}$ 相比, 由 于金属最外层电子数不同 $\left(\mathrm{Ir}\right.$ 为 $\mathrm{d}^{9}$ 金属, 而 $\mathrm{Os}$ 为 $\mathrm{d}^{8}$ 金 属), 导致金属中心在配位环境相同时所带的电荷不同. 比如与双电子配体 CO 配位时, Ir 中心带正电而 Os 中心 不带电; 与单电子配体 $\mathrm{Cl}$ 配位时, $\mathrm{Ir}$ 中心不带电而 $\mathrm{Os}$ 中心带正电.

接下来, 我们对铱杂碳龙配合物 5 和 7 的生成机理 进行了推测. 如 Scheme 5 所示, 在亲核试剂三苯基膦的 作用下, 铱金属源 3 中弱配位的乙腈配体首先发生解离,

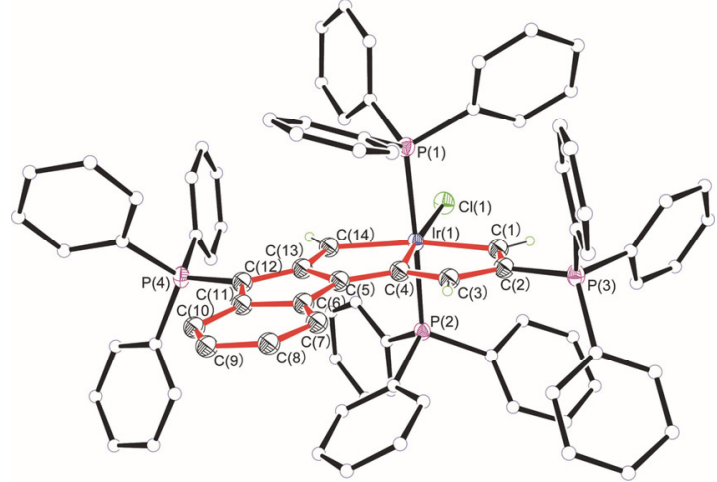

图 4 铱杂碳龙配合物 $\mathbf{6}$ 的阳离子晶体结构

Figure 4 Molecular structure for the cation of irida-carbolong complex 6. Ellipsoids at the $50 \%$ probability level. Counteranion and hydrogen atoms in $\mathrm{PPh}_{3}$ are omitted for clarity.
然后与多炔化合物 2 及三苯基膦反应，生成中间体 Int 1; 随后 Int 1 发生分子内 $[2+2+1]$ 环加成形成中间体 Int 2; 在氟嗍酸水溶液的作用下, Int 2 中与 $\mathrm{C}(3)$ 相连的 羟基离去形成中间体 Int 3; Int 3 中 $\mathrm{C}(12)$ 上的羟基在氟 硼酸水作用下进一步被体系内过量的三苯基膦亲核取 代后形成中间体 Int 4; Int 4 失去一分子氟嗍酸后形成 配合物 5. 在脱羰基试剂二水合氧化三甲胺作用下, $\mathbf{5}$ 可 以失去 $\mathrm{CO}$ 从而形成 16 电子中间体 Int $\mathbf{5}$, 之后再与四 丁基氯化铵反应生成配合物 7.

需要指出的是，由于中间体 Int 2 中的 C(3)和 C(12) 均为手性碳, 理论上它应该包含两对对映异构体. 幸运 的是，通过原位培养单晶的方式我们得到了其中一对异 构体的单晶 $(\mathrm{C}(3)$ 和 $\mathrm{C}(12)$ 上的两个羟基处于反式). 但是 由于还包含其它副产物, Int 2 无法通过核磁等其它手段 被进一步鉴定.

如 Figure 5 所示, Int 2 的主结构仍由四个并环组成, 其中金属被两个五元环所共用. 由键长数据可知, $\mathrm{C}(2)-\mathrm{C}(3)(1.506(6) \AA), \mathrm{C}(3)-\mathrm{C}(4)(1.523(5) \AA)$, $\mathrm{C}(11)-\mathrm{C}(12)(1.510(7) \AA)$ 和 $\mathrm{C}(12)-\mathrm{C}(13)(1.530(6) \AA)$

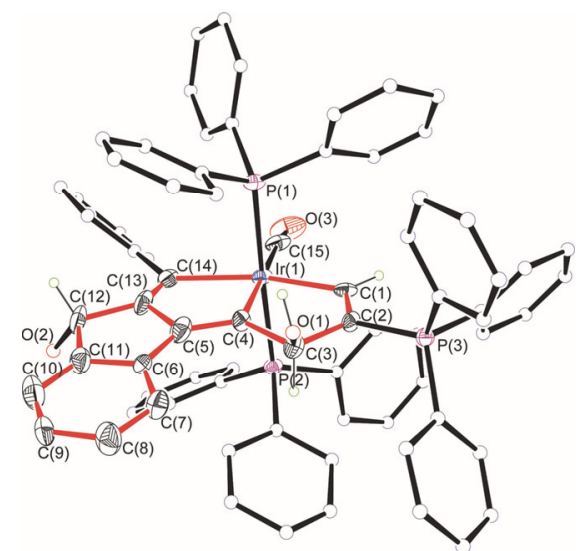

图 5 中间体 Int 2 的阳离子晶体结构

Figure 5 Molecular structure for the cation of intermediate Int 2.

Ellipsoids at the $50 \%$ probability level. Counteranion and hydrogen atoms in $\mathrm{PPh}_{3}$ are omitted for clarity.

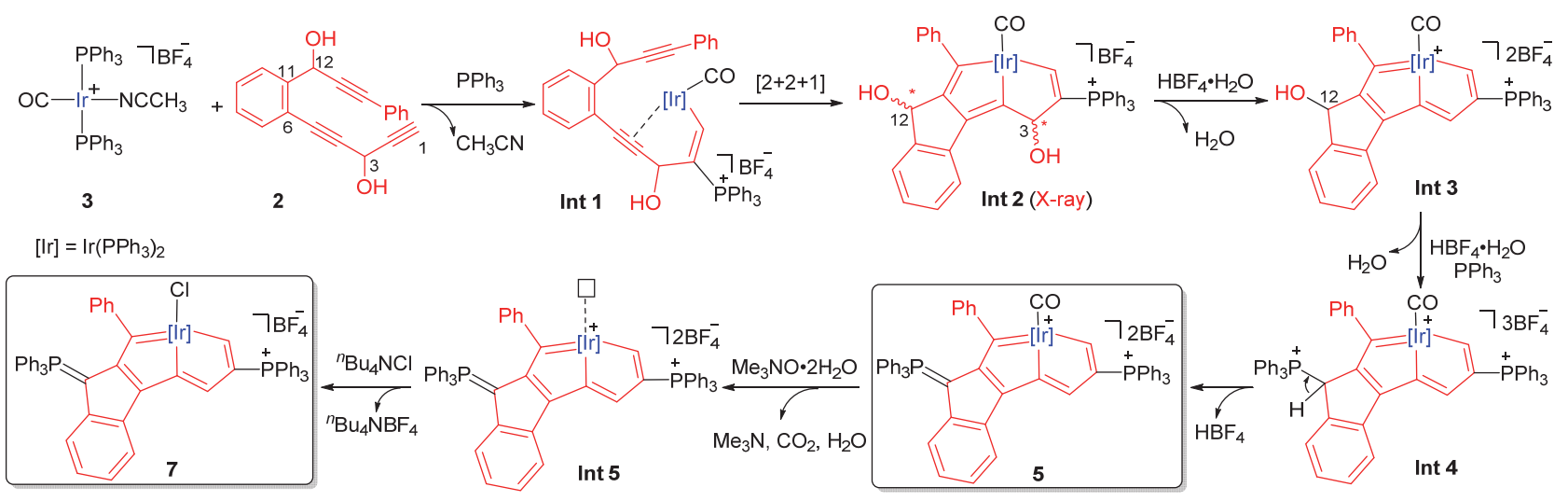

图式 5 铱杂碳龙配合物 5 和 7 的可能生成机理

Scheme 5 The proposed mechanism for the formations of irida-carbolong complexes 5 and 7 
均为典型的 $\mathrm{C}-\mathrm{C}$ 单键; 而 $\mathrm{C}(3)-\mathrm{O}(1)(1.427(5) \AA)$ 以及 $\mathrm{C}(12)-\mathrm{O}(2)(1.442(6) \AA)$ 为 $\mathrm{C}-\mathrm{O}$ 单键, 因此 $\mathrm{C}(3)$ 以及 $\mathrm{C}(12)$ 均为 $\mathrm{sp}^{3}$ 碳. 有趣的是, 尽管 $\mathrm{C}(3)$ 体现出 $\mathrm{sp}^{3}$ 碳的特 性, 但是由 $\operatorname{Ir} 1, C(1) \sim C(5), C(13)$ 和 $C(14)$ 组成的铱杂双 五元环仍具有较好的平面性, 其偏离拟合平面的均方根 偏差仅为 $0.041 \AA$.

基于上述机理, 我们继续研究了配合物 4 和其它配 体的取代反应. 如 Scheme 6 所示, 同样在脱羰基试剂二 水合氧化三甲胺的作用下, 4 与双电子配体环己基异腈 反应可以得到铱杂碳龙配合物 $\mathbf{8}$, 产率为 $85 \%$. 化合物 $\mathbf{8}$ 的单晶结构见 Figure S44.

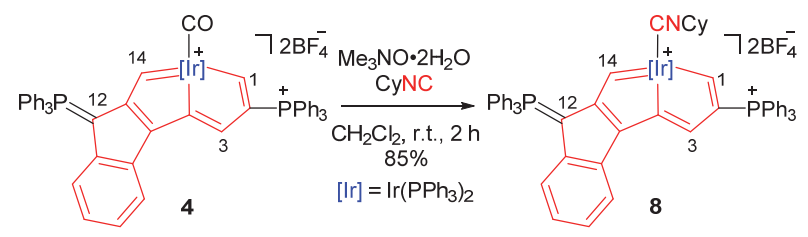

图式 6 铱杂碳龙配合物 8 的合成

Scheme 6 Synthesis of irida-carbolong complex 8

配合物 4 还可以与苯乙炔直接发生反应, 生成金属 中心苯乙炔基取代的铱杂碳龙配合物 $\mathbf{9}$, 产率为 $80 \%$ (Scheme 7).

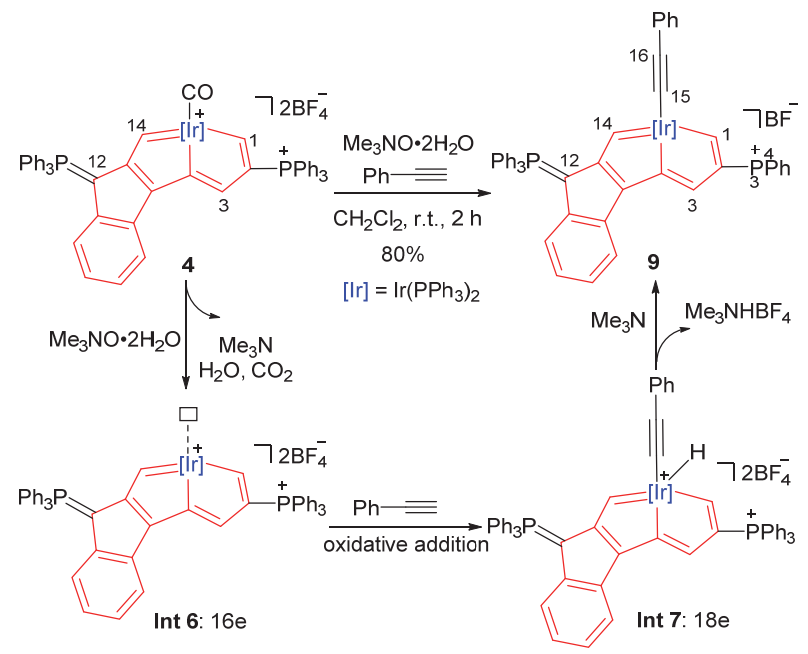

图式 7 铱杂碳龙配合物 9 的合成

Scheme 7 The synthesis of irida-carbolong complex 9

其可能的生成机理如 Scheme 7 所示: 在二水合氧 化三甲胺的作用下, 配合物 4 首先失去 $\mathrm{CO}$, 形成与 Int 5 类似的含有空配位点的 16 电子中间体 Int 6; Int 6 继 续和苯乙炔发生氧化加成反应形成金属氢化物 Int 7 , 此时金属中心满足 18 电子结构; Int 7 可以在原位生成 的三甲胺作用下失去一分子 $\mathrm{HBF}_{4}$ 从而生成最终的产物 9.

在配合物 9 的 ${ }^{1} \mathrm{H}$ NMR 中, 14-H 和 1-H 的信号分别 位于 12.34 和 10.60; 在 ${ }^{13} \mathrm{C}\left\{{ }^{1} \mathrm{H}\right\}$ NMR 中, 14-C 和 1-C 两 个碳原子的化学位移分别位于 218.7 和 189.9 , 它们与铱
杂碳龙配合物 6 的核磁数据类似. 与金属中心配位的苯 乙炔基的两个炔基碳原子的化学位移分别为 116.15 (16-C) 和 99.1 (15-C), 这与文献报道的直接与金属铱配 位的苯乙炔基的两个炔碳化学位移接近 ${ }^{[24]}$. 需要指出 的是, 15-C 因受到金属中心两个膦配体的耦合作用裂分 为三重峰. 在 ${ }^{31} \mathrm{P}\left\{{ }^{1} \mathrm{H}\right\} \mathrm{NMR}$ 中, 金属中心两个等价膦的 化学位移为 -0.89 , 而季鏻信号分别位于 6.24 和 9.98 . 此外, 高分辨质谱表明, 其实验值 1518.4249 与 9 的一价 阳离子的理论值 $\left[\mathrm{C}_{94} \mathrm{H}_{72} \mathrm{IrP}_{4}\right]^{+} 1518.4242$ 吻合, 这进一步 确认了 9 的结构. 苯乙炔基的引入为 9 的后续衍生化提 供了可能性.

\section{4 铱杂碳龙配合物的吸收光谱及光热性能研究}

本文所合成的一系列铱杂碳龙配合物均具有较大 的共轭结构, 因此我们对它们的光物理性质进行了考 察. 首先对铱杂碳龙配合物 $4 \sim 8$ 的紫外-可见-近红外吸 收光谱进行了测试. 如 Figure 6 所示, 以上五种化合物 在紫外-可见区均具有良好的吸收，其中化合物 4,5 和 7 在近红外区也具有一定的吸收. 金属中心的配体对其吸 收会有一定的影响, 比如 4 比 $\mathbf{6}$ 和 8 的吸收更红移, 而 5 也要比 7 的吸收更加红移, 这主要归因于金属中心的 CO 配体具有一定的反馈作用, 可以参与整个体系的共 轭. 值得一提的是, 由于苯基的引入进一步增加了共轭 程度, 因此配合物 5 的红移程度最为明显, 在 800 900 $\mathrm{nm}$ 区域仍具有一定的吸收. 以上结果表明, 通过结构 改性可以很好地调控铱杂碳龙配合物的吸收特性. 化合 物 $4 \sim 8$ 所体现出的宽频吸收特性为后续进行性能研究 提供了丰富的素材.

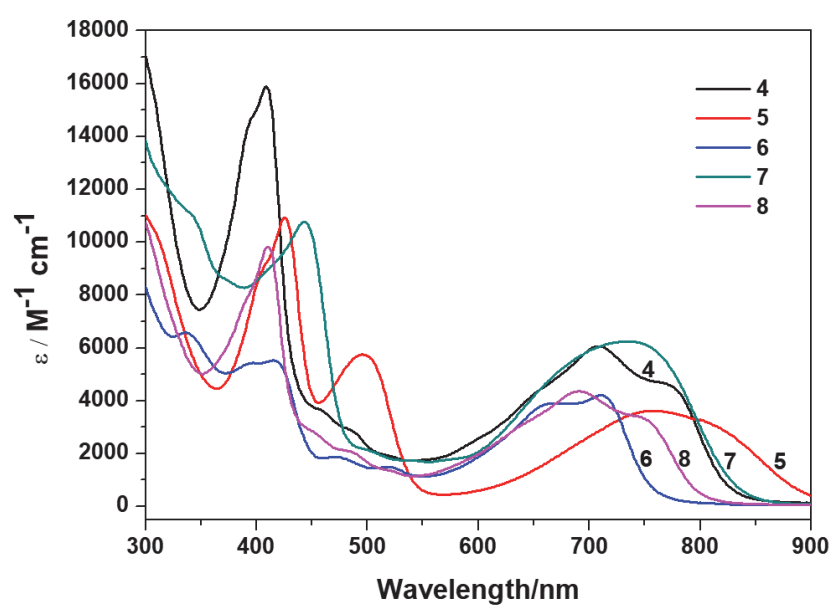

图 6 铱杂碳龙配合物 4 8 的紫外-可见-近红外吸收光谱 Figure 6 UV-Vis-NIR absorption spectra of irida-carbolong complexes $\mathbf{4} \sim \mathbf{8}$, measured in $\mathrm{CH}_{2} \mathrm{Cl}_{2}\left(1.0 \times 10^{-4} \mathrm{~mol} / \mathrm{L}\right)$ at room temperature

由于铱杂碳龙配合物 4 和 $\mathbf{5}$ 在 $808 \mathrm{~nm}$ 附近具有吸 收, 因此我们进一步考察了它们于不同浓度下在 808 $\mathrm{nm}\left(1.0 \mathrm{~W} \cdot \mathrm{cm}^{-2}\right)$ 红外激光照射下的光热效果 ${ }^{[25]}$. 如 Figure 7 所示, 相同浓度下, 化合物 $\mathbf{5}$ 比 $\mathbf{4}$ 具有更好的光 
热效果, 这主要是因为 $\mathbf{5}$ 在 $808 \mathrm{~nm}$ 附近具有更高的摩尔 吸光系数. Figure $7 \mathrm{~b}$ 的结果表明, 含有 $0.1 \mathrm{mg} / \mathrm{mL} 5$ 的乙 醇/水 $\left(V_{\text {乙醇 }} / V_{\text {水 }}=95 / 5\right)$ 在 $808 \mathrm{~nm}$ 的激光照射下, 于 10 $\min$ 内可以使溶液温度升高将近 $40{ }^{\circ} \mathrm{C}$, 而在相同条件 下空白实验的温度则几乎没有变化, 表明 $\mathbf{5}$ 具有良好的 光热效果, 这一现象与本课题组之前报道的相同浓度下 锇杂碳龙配合物的光热效果相当 ${ }^{[13]}$, 为后续基于光热 性能的应用研究提供了可能性. (a)

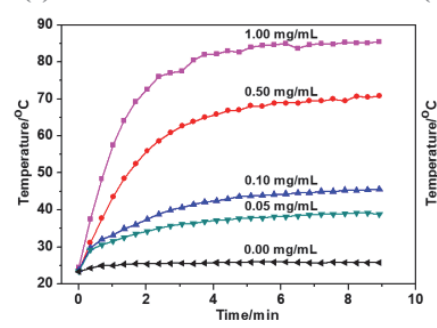

(b)

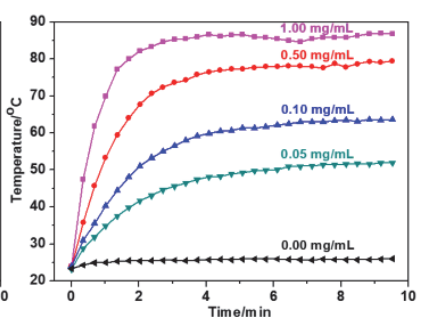

图 7 铱杂碳龙配合物 $\mathbf{4}$ (a) 和 $\mathbf{5}(\mathrm{b})$ 的光热效果

Figure 7 Temperature curves of solutions of irida-carbolong complexes 4 (a) and 5 (b), measured in $\mathrm{EtOH} / \mathrm{H}_{2} \mathrm{O}\left(V(\mathrm{EtOH}) / V\left(\mathrm{H}_{2} \mathrm{O}\right)=95 / 5\right)$ irradiated with an $808 \mathrm{~nm}$ laser at a power density of $1.0 \mathrm{~W} \cdot \mathrm{cm}^{-2}$ at the different concentrations

\section{3 结论}

本文通过两个多炔化合物 $\mathbf{1}$ 和 2 与简单金属铱原料 $\left[\operatorname{Ir}\left(\mathrm{CH}_{3} \mathrm{CN}\right)(\mathrm{CO})\left(\mathrm{PPh}_{3}\right)_{2}\right] \mathrm{BF}_{4}(3)$ 的反应, 一步合成了具有 四个共轭并环结构的铱杂碳龙配合物 4 和 $\mathbf{5}$, 并通过配 体取代反应制备了具有类似结构的铱杂碳龙配合物 6 9. 这不仅是首次通过 “一锅法” 制备含四个共轭并环的 碳龙配合物, 也是首次将碳龙化学拓展至金属铱体系. 光物理性质研究表明, 这些独特的大 $\pi$ 共轭体系在紫外 -可见区具有很好的吸收, 部分甚至在近红外区也表现 出较好的吸收特性; 光热性能研究表明, 化合物 5 具有 良好的光热转化效果. 本文不仅拓展了由多炔有机物与 金属源一锅法构筑碳龙配合物这一策略的适用范围, 而 且丰富了碳龙配合物的种类, 有望为后续开发碳龙配合 物的应用研究提供新素材.

\section{4 实验部分}

如无特别说明, 所有操作都采用标准 Schlenk 操作 并在氮气氛围下进行. 四氢呋喃采用在氮气保护下与钠 丝一起加热回流的方法进行干燥处理. 二氯甲烷采用加 入 $\mathrm{CaH}_{2}$ 加热回流的方法进行干燥处理.

\section{1 化合物 $1 \mathrm{a}$ 的合成}

在 $250 \mathrm{~mL}$ 的反应瓶中, 往 $\mathrm{PdCl}_{2}\left(\mathrm{PPh}_{3}\right)_{2}(5 \mathrm{~mol} \%, 1.9$ $\mathrm{g}), \mathrm{CuI}(5 \mathrm{~mol} \%, 0.5 \mathrm{~g})$ 和邻溴苯甲醛 $(10 \mathrm{~g}, 54.0 \mathrm{mmol})$ 的 混合物中加入干燥 THF $(100 \mathrm{~mL})$, 随后加入炔丙醇 (3.8 mL, $64.9 \mathrm{mmol})$ 和 $\mathrm{Et}_{3} \mathrm{~N}(50 \mathrm{~mL})$, 反应在 $60{ }^{\circ} \mathrm{C}$ 下充 分摚拌过夜. 经 TLC 跟踪反应完全后, 将上述反应液过
滤，固体用 $\operatorname{THF}(2 \times 20 \mathrm{~mL})$ 淋洗，滤液收集后旋干，以 硅胶为固定相，正己烷/乙酸乙酯 $\left(V_{\text {正已㷋 }} / V_{\text {乙酸乙酯 }}=10 / 1\right)$ 为 洗脱剂, 经柱层析分离得到 $6.1 \mathrm{~g}$ 橙黄色油状液体 $1 \mathrm{a}$, 产率为 70\%; ${ }^{1} \mathrm{H} \mathrm{NMR}\left(400.0 \mathrm{MHz}, \mathrm{CDCl}_{3}\right): \delta: 10.46(\mathrm{~d}$, $J(\mathrm{HH})=0.6 \mathrm{~Hz}, 1 \mathrm{H}), 7.84(\mathrm{td}, J(\mathrm{HH})=7.7 \mathrm{~Hz}, J(\mathrm{HH})=$ $0.8 \mathrm{~Hz}, 1 \mathrm{H}), 7.51(\mathrm{~d}, J(\mathrm{HH})=3.8 \mathrm{~Hz}, 2 \mathrm{H}), 7.43 \sim 7.38(\mathrm{~m}$, $1 \mathrm{H}), 4.56(\mathrm{~d}, J(\mathrm{HH})=4.1 \mathrm{~Hz}, 2 \mathrm{H}), 3.12(\mathrm{br}, 1 \mathrm{H}) ;{ }^{13} \mathrm{C}\left\{{ }^{1} \mathrm{H}\right\}$ NMR (100.6 MHz, $\left.\mathrm{CDCl}_{3}\right) \delta: 191.9,135.9,133.8,133.4$, $128.8,127.5,126.1,94.7,81.1,51.4$; HRMS-ESI $(\mathrm{m} / \mathrm{z})$ calcd for $\mathrm{C}_{10} \mathrm{H}_{9} \mathrm{O}_{2}[\mathrm{M}+\mathrm{H}]^{+}$161.0597, found 161.0597.

\section{2 化合物 $1 \mathrm{~b}$ 的合成}

在 $100 \mathrm{~mL}$ 的圆底烧瓶中加入 $1 \mathrm{a}(3.2 \mathrm{~g}, 20.0 \mathrm{mmol})$ 和 2-碘酰基苯甲酸 $(6.7 \mathrm{~g}, 24.0 \mathrm{mmol})$, 随后加入 $50 \mathrm{~mL}$ DMSO, 在室温和空气氛下搅拌 $2 \mathrm{~h}$, 经 TLC 跟踪反应 完全后, 加入 $50 \mathrm{~mL}$ 水猝灭反应, 在该过程中伴随着沉 淀析出和放热, 待恢复至室温后, 将沉淀过滤并用乙酸 乙酯淋洗 $(2 \times 20 \mathrm{~mL})$ ，合并滤液，用乙酸乙酯 $(3 \times 200$ $\mathrm{mL})$ 萃取, 饱和 $\mathrm{NaCl}$ 溶液 $(3 \times 100 \mathrm{~mL})$ 洗涤, 有机相经 无水硫酸镁干燥后旋干. 然后以硅胶为固定相, 正己烷/ 乙酸乙酯 $\left(V_{\text {正已㟋 }} / V_{\text {乙酸乙酟 }}=10 / 1\right)$ 为洗脱剂, 经柱层析分离 得到 $2.0 \mathrm{~g}$ 橙黄色固体 1b, 产率为 $62 \% ;{ }^{1} \mathrm{H}$ NMR (400.0 $\left.\mathrm{MHz}, \mathrm{CDCl}_{3}\right) \delta: 10.48(\mathrm{~s}, 1 \mathrm{H}), 9.49(\mathrm{~s}, 1 \mathrm{H}), 8.00 \sim 7.98$ $(\mathrm{m}, 1 \mathrm{H}), 7.74 \sim 7.72(\mathrm{~m}, 1 \mathrm{H}) 7.69 \sim 7.62(\mathrm{~m}, 2 \mathrm{H}) ;{ }^{13} \mathrm{C}\left\{{ }^{1} \mathrm{H}\right\}$ NMR (100.6 MHz, $\left.\mathrm{CDCl}_{3}\right): \delta: 190.0,176.2,137.2,134.8$, 133.9, 131.5, 128.3, 122.0, 93.1, 89.6; HRMS-ESI $(\mathrm{m} / \mathrm{z})$ calcd for $\mathrm{C}_{10} \mathrm{H}_{6} \mathrm{NaO}_{2}[\mathrm{M}+\mathrm{H}]^{+}$159.0441, found 159.0442 .

\section{3 化合物 1c 的合成}

在经过高温烘烤的 $200 \mathrm{~mL}$ 反应瓶中加入三甲基硅 基乙炔(3.9 ml, $27.7 \mathrm{mmol}$ ), 并用 $50 \mathrm{~mL}$ THF 稀释，随后 在-78 ${ }^{\circ} \mathrm{C}$ 下用注射泵于 $40 \mathrm{~min}$ 内缓慢滴加正丁基锂 (12.6 mL, 2.5 M in THF, $31.5 \mathrm{mmol}$ ). 滴加结束后在该温 度下继续搅拌 $20 \mathrm{~min}$, 然后用注射葲将含有 $\mathbf{1 b}(2.0 \mathrm{~g}$, $12.6 \mathrm{mmol})$ 的 $\mathrm{THF}(20 \mathrm{~mL})$ 溶液缓慢加入上述反应体系 中. 经 TLC 跟踪反应, $2 \mathrm{~h}$ 后反应结束, 然后用饱和 $\mathrm{NH}_{4} \mathrm{Cl}(50 \mathrm{~mL})$ 淬灭反应. 待恢复至室温后, 用乙酸乙酯 $(3 \times 100 \mathrm{~mL})$ 萃取, 饱和 $\mathrm{NaCl}$ 溶液 $(3 \times 100 \mathrm{~mL})$ 洗涤, 有机相经无水硫酸镁干燥后旋干. 然后以硅胶为固定 相, 正己烷/乙酸乙酯 $(V$ 正已䏓 $/ V$ 乙酸乙酯 $=5 / 1)$ 为洗脱剂, 经 柱层析分离得到 $2.6 \mathrm{~g}$ 棕黄色油状物 1c, 产率为 $60 \% ;{ }^{1} \mathrm{H}$ NMR (400.0 MHz, $\left.\mathrm{CDCl}_{3}\right) \delta: 7.74(\mathrm{~d}, J(\mathrm{HH})=7.6 \mathrm{~Hz}$, $1 \mathrm{H}), 7.45(\mathrm{t}, J(\mathrm{HH})=7.4 \mathrm{~Hz}, 1 \mathrm{H}), 7.36(\mathrm{t}, J(\mathrm{HH})=7.6 \mathrm{~Hz}$, $1 \mathrm{H}), 7.27(\mathrm{t}, J(\mathrm{HH})=7.4 \mathrm{~Hz}, 1 \mathrm{H}), 5.86(\mathrm{~d}, J(\mathrm{HH})=9.5 \mathrm{~Hz}$, $1 \mathrm{H}), 5.37(\mathrm{~d}, J(\mathrm{HH})=9.5 \mathrm{~Hz}, 1 \mathrm{H}), 4.10(\mathrm{br}, 1 \mathrm{H}), 3.58(\mathrm{br}$, $1 \mathrm{H}), 0.22\left(\mathrm{~s}, 18 \mathrm{H}, \mathrm{C}\left(\mathrm{CH}_{3}\right)_{3}\right) ;{ }^{13} \mathrm{C}\left\{{ }^{1} \mathrm{H}\right\} \mathrm{NMR}(100.6 \mathrm{MHz}$, $\left.\mathrm{CDCl}_{3}\right) \delta: 141.9,132.4,129.1,128.2,126.9,120.8,104.2$, $101.7,91.7,91.4,89.5,81.6,62.9,52.7,0.3$; HRMS-ESI $(m / z)$ calcd for $\mathrm{C}_{20} \mathrm{H}_{26} \mathrm{O}_{2} \mathrm{Si}_{2} \mathrm{Na}[\mathrm{M}+\mathrm{Na}]^{+} 377.1364$, found 
377.1366.

\section{4 多炔化合物 1 的合成}

在 $100 \mathrm{~mL}$ 的圆底烧瓶中加入 $\mathbf{1 c}(2.6 \mathrm{~g}, 7.3 \mathrm{mmol})$ 并用 $50 \mathrm{~mL} \mathrm{THF}$ 稀释, 然后在 $-40{ }^{\circ} \mathrm{C}$ 下加入四丁基氯 化铵的四氢呋喃溶液 $(1.0 \mathrm{M}$ in $\mathrm{THF}, 17.6 \mathrm{~mL}, 17.6$ $\mathrm{mmol})$. 空气氛中室温搅拌 $30 \mathrm{~min}$ 后, 经 TLC 跟踪发现 反应完全, 然后加入饱和 $\mathrm{NH}_{4} \mathrm{Cl}$ 溶液 $(50 \mathrm{~mL}$ ) 淬灭反 应. 经乙酸乙酯 $(3 \times 100 \mathrm{~mL})$ 萃取, 饱和 $\mathrm{NaCl}$ 溶液 $(3 \times$ $50 \mathrm{~mL}$ ) 洗涤, 有机相经无水硫酸镁干燥后旋干. 然后以

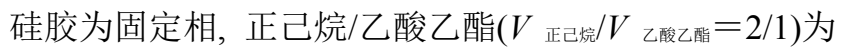
洗脱剂, 经柱层析分离得到 $1.4 \mathrm{~g}$ 黄色油状物 $\mathbf{1}$, 产率为 93\%; ${ }^{1} \mathrm{H}$ NMR (400.0 MHz, $\left.\mathrm{CDCl}_{3}\right) \delta: 7.74(\mathrm{dd}, J(\mathrm{HH})=$ $7.4 \mathrm{~Hz}, J(\mathrm{HH})=2.9 \mathrm{~Hz}, 1 \mathrm{H}), 7.49 \sim 7.46(\mathrm{~m}, 1 \mathrm{H}), 7.41 \sim$ $7.36(\mathrm{~m}, 1 \mathrm{H}), 7.31 \sim 7.27(\mathrm{~m}, 1 \mathrm{H}), 5.86(\mathrm{~d}, J(\mathrm{HH})=9.5$ $\mathrm{Hz}, 1 \mathrm{H}), 5.38$ (br, 1H), 4.22 (br, 1H), 3.67 (br, 1H), 2.67 $(\mathrm{dd}, J(\mathrm{HH})=2.2 \mathrm{~Hz}, J(\mathrm{HH})=1.2 \mathrm{~Hz}, 1 \mathrm{H}), 2.63(\mathrm{t}, J(\mathrm{HH})$ $=2.1 \mathrm{~Hz}, 1 \mathrm{H}) ;{ }^{13} \mathrm{C}\left\{{ }^{1} \mathrm{H}\right\} \mathrm{NMR}\left(100.6 \mathrm{MHz}, \mathrm{CDCl}_{3}\right) \delta$ : $141.6,132.7,129.5,128.5,126.9,120.5,91.0,82.6,81.8$, 80.6, 75.3, 73.2, 62.6, 52.3; HRMS-ESI $(\mathrm{m} / \mathrm{z})$ calcd for $\mathrm{C}_{14} \mathrm{H}_{10} \mathrm{O}_{2} \mathrm{Na}[\mathrm{M}+\mathrm{H}]^{+}$211.0754, found 211.0755. 需要指 出的是, 该化合物存在两对对映异构体, 其物质的量比 例大约为 $1: 1$.

\section{5 化合物 $2 \mathrm{a}$ 的合成}

在 $250 \mathrm{~mL}$ 的反应瓶中, 往 $\mathrm{PdCl}_{2}\left(\mathrm{PPh}_{3}\right)_{2}(5 \mathrm{~mol} \%, 1.9$ $\mathrm{g}) 、 \mathrm{CuI}(5 \mathrm{~mol} \%, 0.5 \mathrm{~g})$ 和邻溴苯甲醛 $(10 \mathrm{~g}, 54.0 \mathrm{mmol})$ 的 混合物中加入干燥 $\mathrm{THF}(100 \mathrm{~mL})$, 随后加入三甲基硅基 乙炔 $(9.2 \mathrm{~mL}, 64.8 \mathrm{mmol})$ 和 $\mathrm{Et}_{3} \mathrm{~N}(50 \mathrm{~mL})$, 反应在 $60{ }^{\circ} \mathrm{C}$ 下充分摚拌过夜. 经 TLC 跟踪反应完全后, 将上述反应 液过滤，固体用 $\mathrm{THF}(2 \times 20 \mathrm{~mL})$ 淋洗，滤液收集后旋干，

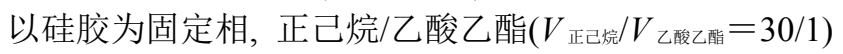
为洗脱剂, 经柱层析分离得到 $10.4 \mathrm{~g}$ 橙黄色固体 1a, 产 率为 95\%; ${ }^{1} \mathrm{H} \mathrm{NMR}\left(400.0 \mathrm{MHz}, \mathrm{CDCl}_{3}\right) \delta: 10.56(\mathrm{~s}, 1 \mathrm{H})$, $7.91(\mathrm{~d}, J(\mathrm{HH})=7.8 \mathrm{~Hz}, 1 \mathrm{H}), 7.56 \sim 7.54(\mathrm{~m}, 2 \mathrm{H}), 7.43(\mathrm{t}$, $J(\mathrm{HH})=7.8 \mathrm{~Hz}, 1 \mathrm{H}), 0.29\left(\mathrm{~s}, 9 \mathrm{H}, \mathrm{C}\left(\mathrm{CH}_{3}\right)_{3}\right) ;{ }^{13} \mathrm{C}\left\{{ }^{1} \mathrm{H}\right\} \mathrm{NMR}$ $\left(100.6 \mathrm{MHz}, \mathrm{CDCl}_{3}\right) \delta: 191.8,136.2,133.7,133.5,128.8$, $126.9,126.7,102.4,100.1,-0.2$. 化合物 $2 \mathbf{a}$ 的核磁数 据与文献一致 ${ }^{[26]}$.

\section{6 化合物 $\mathbf{2 b}$ 的合成}

在经过高温烘烤的 $500 \mathrm{~mL}$ 反应瓶中加入 $2 \mathrm{a}(8.8 \mathrm{~g}$, $43.5 \mathrm{mmol}$ ), 并用 $200 \mathrm{~mL} \mathrm{THF}$ 稀释, 随后在 $-78{ }^{\circ} \mathrm{C}$ 下 用注射百于 $40 \mathrm{~min}$ 内缓慢加入苯乙炔基锂(52.2 mL, 1.0 $\mathrm{M}$ in THF, $31.6 \mathrm{mmol}$ ). 加完后在该温度下继续搅拌 20 $\min$ 后恢复至室温, 反应 $1 \mathrm{~h}$ 后经 TLC 跟踪反应完全. 用饱和 $\mathrm{NH}_{4} \mathrm{Cl}$ 溶液 $(100 \mathrm{~mL})$ 淬灭反应, 用乙醚 $(3 \times 100$ $\mathrm{mL})$ 萃取, 饱和 $\mathrm{NaCl}$ 溶液 $(3 \times 100 \mathrm{~mL})$ 洗涤, 有机相经 无水硫酸镁干燥后旋干. 然后以硅胶为固定相, 正己烷/ 乙酸乙酯 $\left(V_{\text {正已㟋 }} / V_{\text {乙酸乙酯 }}=30 / 1\right)$ 为洗脱剂, 经柱层析分离
得到 $10.7 \mathrm{~g}$ 黄色油状物 $\mathbf{2 b}$, 产率为 $81 \%$; ${ }^{1} \mathrm{H} \mathrm{NMR}$ $\left(500.1 \mathrm{MHz}, \mathrm{CDCl}_{3}\right) \delta: 7.76(\mathrm{~d}, J(\mathrm{HH})=7.9 \mathrm{~Hz}, 1 \mathrm{H}), 7.54$ $(\mathrm{dd}, J(\mathrm{HH})=7.6 \mathrm{~Hz}, J(\mathrm{HH})=1.3 \mathrm{~Hz}, 1 \mathrm{H}), 7.51 \sim 7.49(\mathrm{~m}$, $2 \mathrm{H}), 7.41(\mathrm{t}, J(\mathrm{HH})=7.8 \mathrm{~Hz}, 1 \mathrm{H}), 7.34 \sim 7.31(\mathrm{~m}, 4 \mathrm{H})$, $6.09(\mathrm{~d}, J(\mathrm{HH})=5.9 \mathrm{~Hz}, 1 \mathrm{H}, \mathrm{CH}(\mathrm{OH})), 3.07(\mathrm{~d}, J(\mathrm{HH})=$ $5.9 \mathrm{~Hz}, 1 \mathrm{H}, \mathrm{CH}(\mathrm{OH})), 0.31\left(\mathrm{~s}, 9 \mathrm{H}, \mathrm{C}\left(\mathrm{CH}_{3}\right)_{3}\right) ;{ }^{13} \mathrm{C}\left\{{ }^{1} \mathrm{H}\right\}$ NMR (125.8 MHz, $\left.\mathrm{CDCl}_{3}\right) \delta: 142.9,132.8,131.8,129.2$, $128.5,128.3,128.2,126.7,122.6,121.2,102.4,100.8$, $88.2,88.5,63.8,-0.1$. 化合物 $\mathbf{2 b}$ 的核磁数据与文献一 致 $^{[27]}$.

\section{7 化合物 $2 \mathrm{c}$ 的合成}

在 $200 \mathrm{~mL}$ 的圆底烧瓶中加入 $2 \mathbf{b}(6.8 \mathrm{~g}, 22.3 \mathrm{mmol})$ 并用 $100 \mathrm{~mL}$ THF 稀释, 然后在 $0{ }^{\circ} \mathrm{C}$ 下加入四丁基氯化 铵的四氢呋喃溶液 (1.0 M in THF, $26.8 \mathrm{~mL}, 26.8 \mathrm{mmol}$ ), 空气氛中常温搅拌 $30 \mathrm{~min}$, 经 TLC 跟踪反应完全后, 加 入饱和 $\mathrm{NH}_{4} \mathrm{Cl}$ 溶液 $(100 \mathrm{~mL})$ 淬灭反应, 经乙醚 $(3 \times 100$ $\mathrm{mL})$ 萃取, 再用饱和 $\mathrm{NaCl}$ 溶液 $(3 \times 50 \mathrm{~mL})$ 洗涤, 有机相 经无水硫酸镁干燥后旋干. 然后以硅胶为固定相, 正己 烷/乙酸乙酯 $\left(V_{\text {正已䏓 }} / V_{\text {乙酸乙酯 }}=10 / 1\right)$ 为洗脱剂, 经柱层析分 离得到 $5.0 \mathrm{~g}$ 黄色油状物 $2 \mathrm{c}$, 产率为 $96 \%$; ${ }^{1} \mathrm{H} \mathrm{NMR}$ $\left(500.1 \mathrm{MHz}, \mathrm{CDCl}_{3}\right) \delta: 7.83(\mathrm{~d}, J(\mathrm{HH})=7.1 \mathrm{~Hz}, 1 \mathrm{H}), 7.58$ $(\mathrm{dd}, J(\mathrm{HH})=7.6 \mathrm{~Hz}, J(\mathrm{HH})=1.1 \mathrm{~Hz}, 1 \mathrm{H}), 7.52 \sim 7.49(\mathrm{~m}$, $2 \mathrm{H}), 7.45(\mathrm{t}, J(\mathrm{HH})=7.6 \mathrm{~Hz}, 1 \mathrm{H}), 7.35 \sim 7.32(\mathrm{~m}, 4 \mathrm{H})$, $6.14(\mathrm{~d}, J(\mathrm{HH})=4.4 \mathrm{~Hz}, 1 \mathrm{H}, \mathrm{CH}(\mathrm{OH})), 3.44(\mathrm{~s}, 1 \mathrm{H}, \mathrm{CCH})$, $2.90(\mathrm{~d}, J(\mathrm{HH})=4.4 \mathrm{~Hz}, 1 \mathrm{H}, \mathrm{CH}(\mathrm{OH})) ;{ }^{13} \mathrm{C}\left\{{ }^{1} \mathrm{H}\right\} \mathrm{NMR}$ $\left(125.8 \mathrm{MHz} \mathrm{CDCl}_{3}\right) \delta: 142.9,132.2,131.8,129.5,128.6$, $128.3,126.9,122.5,120.5,88.2,88.6,82.8,81.1,63.5$. 化 合物 $2 \mathrm{c}$ 的核磁数据与文献一致 ${ }^{[28]}$.

\section{8 化合物 $\mathbf{2 d}$ 的合成}

在经过高温烘烤的 $200 \mathrm{~mL}$ 反应瓶中加入 $2 \mathbf{c}(9.3 \mathrm{~g}$, $40.0 \mathrm{mmol}$ ), 并用 $200 \mathrm{~mL} \mathrm{THF}$ 稀释, 随后在氮气氛、 $-40{ }^{\circ} \mathrm{C}$ 下用注射泵于 $1 \mathrm{~h}$ 内缓慢加入二异丙基氨基锂 (2.0 M in THF, $50 \mathrm{~mL}, 100.0 \mathrm{mmol}$ ). 加完后在该温度下 继续搅拌 $1 \mathrm{~h}$ 后升至 $0{ }^{\circ} \mathrm{C}$ 反应过夜. 经 TLC 跟踪反应 完全, 用饱和 $\mathrm{NH}_{4} \mathrm{Cl}$ 溶液 $(100 \mathrm{~mL})$ 淬灭反应, 用乙酸乙 酯 $(3 \times 100 \mathrm{~mL})$ 萃取, 再用饱和 $\mathrm{NaCl}$ 溶液 $(3 \times 100 \mathrm{~mL})$ 洗涤, 有机相经无水硫酸镁干燥后旋干. 然后以硅胶为 固定相, 正己烷/乙酸乙酯 $\left(V_{\text {正已㟋 }} / V_{\text {乙酸乙酯 }}=5 / 1\right)$ 为洗脱剂, 经柱层析分离得到 $8.7 \mathrm{~g}$ 棕黄色油状物 2d, 产率为 $61 \%$; ${ }^{1} \mathrm{H}$ NMR $\left(500.1 \mathrm{MHz}, \mathrm{CDCl}_{3}\right) \delta: 7.81(\mathrm{~d}, J(\mathrm{HH})=7.7 \mathrm{~Hz}$, $1 \mathrm{H}), 7.53 \sim 7.49(\mathrm{~m}, 3 \mathrm{H}), 7.43 \sim 7.40(\mathrm{~m}, 2 \mathrm{H}), 7.34 \sim 7.31$ $(\mathrm{m}, 3 \mathrm{H}), 6.09(\mathrm{~d}, J(\mathrm{HH})=4.2 \mathrm{~Hz}, 1 \mathrm{H}), 5.40(\mathrm{~d}, J(\mathrm{HH})=$ $4.2 \mathrm{~Hz}, 1 \mathrm{H}), 3.39$ (br, 1H), 3.28 (br, 1H), 0.24 (s, 9H); ${ }^{13} \mathrm{C}\left\{{ }^{1} \mathrm{H}\right\}$ NMR (125.8 MHz, $\left.\mathrm{CDCl}_{3}\right) \delta: 142.8,132.7,131.8$, $129.4,128.6,128.3,127.0,123.9,122.5,120.6,120.5$, 101.7, 91.7, 90.1, 88.2, 86.8, 81.9, 63.5, 53.1, - 0.3; HRMS-ESI $(m / z)$ calcd for $\mathrm{C}_{23} \mathrm{H}_{22} \mathrm{O}_{2} \mathrm{SiNa}[\mathrm{M}+\mathrm{Na}]^{+}$ 381.1281 , found 381.1285 . 


\section{9 多炔化合物 2 的合成}

在 $200 \mathrm{~mL}$ 的圆底烧瓶中加入 $2 \mathbf{d}(8.7 \mathrm{~g}, 24.3 \mathrm{mmol})$ 并用 $100 \mathrm{~mL} \mathrm{THF}$ 稀释, 然后在 $-40{ }^{\circ} \mathrm{C}$ 下加入四丁基氯 化铵的四氢呋喃溶液(1.0 M in THF, $29.2 \mathrm{~mL}, 29.2$ $\mathrm{mmol})$, 常温、空气氛中搅拌 $30 \mathrm{~min}$, 经 TLC 跟踪反应 完全后, 加入饱和 $\mathrm{NH}_{4} \mathrm{Cl}$ 溶液 $(100 \mathrm{~mL})$ 淬灭反应, 经乙 酸乙酯 $(3 \times 100 \mathrm{~mL})$ 萃取, 饱和 $\mathrm{NaCl}$ 溶液 $(3 \times 50 \mathrm{~mL})$ 洗 涤, 有机相经无水硫酸镁干燥后旋干. 然后以硅胶为固 定相, 正己烷/乙酸乙酯 $(V$ 正已㟋 $/ V$ 乙酸乙酯 $=3 / 1)$ 为洗脱剂, 经柱层析分离得到 $5.9 \mathrm{~g}$ 棕色油状物 2, 产率为 $85 \% ;{ }^{1} \mathrm{H}$ NMR (500.1 MHz, $\left.\mathrm{CDCl}_{3}\right) \delta: 7.83(\mathrm{~d}, J(\mathrm{HH})=7.4 \mathrm{~Hz}$, $1 \mathrm{H}), 7.49 \sim 7.48(\mathrm{~m}, 3 \mathrm{H}), 7.39(\mathrm{t}, J(\mathrm{HH})=7.6 \mathrm{~Hz}, 2 \mathrm{H})$, $7.34 \sim 7.28(\mathrm{~m}, 3 \mathrm{H}), 6.11(\mathrm{~d}, J(\mathrm{HH})=12.9 \mathrm{~Hz}, 1 \mathrm{H}), 5.40$ $(\mathrm{d}, J(\mathrm{HH})=9.2 \mathrm{~Hz}, 1 \mathrm{H}), 4.33,4.24(\mathrm{br}, 1 \mathrm{H}), 3.75(\mathrm{br}, 1 \mathrm{H})$, $2.61(\mathrm{~d}, J(\mathrm{HH})=2.3 \mathrm{~Hz}, 1 \mathrm{H}) ;{ }^{13} \mathrm{C}\left\{{ }^{1} \mathrm{H}\right\} \mathrm{NMR}(125.8 \mathrm{MHz}$, $\left.\mathrm{CDCl}_{3}\right) \delta: 142.4,132.8,131.8,129.5,128.6,128.4,128.3$, $127.1,122.5,120.6,120.5,91.3,88.1,86.9,81.9,80.8$, $80.7,73.1,63.4,52.4$; HRMS-ESI $(\mathrm{m} / \mathrm{z})$ calcd for $\mathrm{C}_{20} \mathrm{H}_{14} \mathrm{O}_{2} \mathrm{Na}[\mathrm{M}+\mathrm{Na}]^{+}$309.0886, found 309.0884. 需要 指出的是, 该化合物存在两对对映异构体, 其比例大约 为 $5: 3$.

\subsection{0 铱原料 $\left[\operatorname{Ir}\left(\mathrm{CH}_{3} \mathrm{CN}\right)(\mathrm{CO})\left(\mathrm{PPh}_{3}\right)_{2}\right] \mathrm{BF}_{4}(3)$ 的合成}

在 $200 \mathrm{~mL}$ 的圆底烧瓶中加入 Vaska's 络合物 $\operatorname{IrCl}(\mathrm{CO})\left(\mathrm{PPh}_{3}\right)_{2}(1.0 \mathrm{~g}, 1.28 \mathrm{mmol})$ 和四氟硼酸银 $(275 \mathrm{mg}$, $1.41 \mathrm{mmol})$, 随后加入 $100 \mathrm{~mL}$ 乙腈溶解, 在常温下反应 $40 \mathrm{~min}$. 待反应结束后, 用硅藻土滤去银盐, 滤液旋 干 ${ }^{[7 a]}$. 然后以硅胶为固定相, 丙酮 $/$ 二氯甲烷 $\left(V_{\text {丙醐 }} / V_{\text {二氯甲烷 }}\right.$ $=1 / 10$ 为洗脱剂, 经柱层析分离得到 $950 \mathrm{mg}$ 黄色固体 3, 产率为 $85 \%$.

\subsection{1 铱杂碳龙配合物 4 的合成}

在 $50 \mathrm{~mL}$ 的反应瓶中加入铱原料 $3(1.00 \mathrm{~g}, 1.15$ $\mathrm{mmol}$ ) 和三苯基膦 $(900 \mathrm{mg}, 3.44 \mathrm{mmol})$, 用 $30 \mathrm{~mL}$ 二氯 甲烷溶解, 随后加入多炔化合物 1(364 mg, $1.73 \mathrm{mmol})$ 的二氯甲烷 $(5 \mathrm{~mL})$ 溶液. 在常温下搅拌 $30 \mathrm{~min}$ 后得到红 色溶液, 之后加入氟硼酸水溶液 $(366 \mu \mathrm{L}, 5.75 \mathrm{mmol})$ 立 即变成红色, 继续反应 $20 \mathrm{~min}$. 将反应液浓缩、乙醚洗 涤 $(3 \times 20 \mathrm{~mL})$ 和真空干燥后得到 $1.58 \mathrm{~g}$ 绿色固体 4, 产 率为 $85 \%$. ${ }^{1} \mathrm{H}$ NMR $\left(500.1 \mathrm{MHz}, \mathrm{CD}_{2} \mathrm{Cl}_{2}\right) \delta: 10.62(\mathrm{~s}, 1 \mathrm{H}$, $\mathrm{C} 14 H), 9.80(\mathrm{~d}, J(\mathrm{PH})=21.5 \mathrm{~Hz}, 1 \mathrm{H}, \mathrm{C} 1 H), 8.42(\mathrm{~s}, 1 \mathrm{H}$, $\mathrm{C} 3 H), 7.94 \sim 6.34(64 \mathrm{H}, \mathrm{Ph}) ;{ }^{31} \mathrm{P}\left\{{ }^{1} \mathrm{H}\right\}$ NMR $(202.5 \mathrm{MHz}$, $\left.\mathrm{CD}_{2} \mathrm{Cl}_{2}\right) \delta: 13.45\left(\mathrm{~s}, \mathrm{CPPh}_{3}\right), 6.66\left(\mathrm{~s}, \mathrm{C} P \mathrm{Ph}_{3}\right), 1.98(\mathrm{~s}$, $\left.\mathrm{Ir} P P h_{3}\right) ;{ }^{13} \mathrm{C}\left\{{ }^{1} \mathrm{H}\right\} \quad \mathrm{NMR} \quad\left(125.8 \mathrm{MHz}, \mathrm{CD}_{2} \mathrm{Cl}_{2}\right.$, plus 13C-dept $135,{ }^{1} \mathrm{H}-{ }^{13} \mathrm{C}$ HSQC and ${ }^{1} \mathrm{H}-{ }^{13} \mathrm{C}$ HMBC) $\delta: 195.2$ (br, C14), 177.5 (br, C1), $174.6(\mathrm{t}, J(\mathrm{PC})=9.2 \mathrm{~Hz}, \mathrm{C} 15)$, $173.6(\mathrm{~d}, J(\mathrm{PC})=13.4 \mathrm{~Hz}, \mathrm{C} 13), 170.3(\mathrm{dt}, J(\mathrm{PC})=24.1$ $\mathrm{Hz}, J(\mathrm{PC})=4.1 \mathrm{~Hz}, \mathrm{C} 4), 158.2(\mathrm{~d}, J(\mathrm{PC})=10.8 \mathrm{~Hz}, \mathrm{C} 5)$, $139.4(\mathrm{~d}, J(\mathrm{PC})=24.0 \mathrm{~Hz}, \mathrm{C} 3), 121.8(\mathrm{~d}, J(\mathrm{PC})=90.9 \mathrm{~Hz}$,
C2), $135.5 \sim 119.3(\mathrm{Ph}), 73.5(\mathrm{~d}, J(\mathrm{PC})=118.2 \mathrm{~Hz}, \mathrm{C} 12)$; IR $v$ : $2019(\mathrm{CO}) \mathrm{cm}^{-1}$; HRMS-ESI $(\mathrm{m} / \mathrm{z})$ calcd for $\left[\mathrm{C}_{87} \mathrm{H}_{67} \mathrm{IrOP}_{4}\right]^{2+}$ 722.1880, found, 722.1885.

\subsection{2 铱杂碳龙配合物 5 的合成}

在 $50 \mathrm{~mL}$ 的反应瓶中加入铱原料 $\mathbf{3}(500 \mathrm{mg}, 0.57$ $\mathrm{mmol}$ )和三苯基膦(448 mg, $1.71 \mathrm{mmol})$, 用 $15 \mathrm{~mL}$ 二氯 甲烷溶解，随后加入多炔化合物 $2(196 \mathrm{mg}, 0.68 \mathrm{mmol}$ ) 的二氯甲烷 $(5 \mathrm{~mL})$ 溶液. 在常温下搅拌 $60 \mathrm{~min}$ 后得到绿 色溶液, 之后加入氟硼酸水溶液 $(180 \mu \mathrm{L}, 2.85 \mathrm{mmol})$ 立 即变成黄绿色, 继续反应 $30 \mathrm{~min}$. 将反应液浓缩, 经中 性氧化铝柱色谱(洗脱剂: $V$ 二氯甲烷 $/ V$ 丙䣩 $=5 / 1$ ) 分离得到 $603 \mathrm{mg}$ 黄绿色固体 5, 产率为 65\%. ${ }^{1} \mathrm{H}$ NMR (600.1 $\left.\mathrm{MHz}, \mathrm{CD}_{2} \mathrm{Cl}_{2}\right) \delta$ : $9.75(\mathrm{~d}, J(\mathrm{PH})=20.2 \mathrm{~Hz}, 1 \mathrm{H}, \mathrm{C} 1 H), 8.41$ $(\mathrm{s}, 1 \mathrm{H}, \mathrm{C} 3 H), 7.94 \sim 6.19(69 \mathrm{H}, \mathrm{Ph}$ and $\mathrm{C} 7 H-\mathrm{C} 10 H)$; ${ }^{31} \mathrm{P}\left\{{ }^{1} \mathrm{H}\right\}$ NMR $\left(242.9 \mathrm{MHz}, \mathrm{CD}_{2} \mathrm{Cl}_{2}\right) \delta: 12.32(\mathrm{~s}, \mathrm{CPPh})$, $7.49(\mathrm{~s}, \mathrm{CPPh}),-6.80\left(\mathrm{~s}, \operatorname{Ir} P \mathrm{Ph}_{3}\right) ;{ }^{13} \mathrm{C}\left\{{ }^{1} \mathrm{H}\right\}$ NMR (150.9 $\mathrm{MHz}, \mathrm{CD}_{2} \mathrm{Cl}_{2}$, plus ${ }^{13} \mathrm{C}$-dept $135,{ }^{1} \mathrm{H}^{13} \mathrm{C}$ HSQC and ${ }^{1} \mathrm{H}_{-}{ }^{13} \mathrm{C}$ HMBC): $\delta 212.3$ (br, C14), 175.9 (br, C1), 175.7 (t, $J(\mathrm{PC})=8.9 \mathrm{~Hz}, \mathrm{C} 15), 170.8(\mathrm{~d}, J(\mathrm{PC})=10.6 \mathrm{~Hz}, \mathrm{C} 13)$, $166.1(\mathrm{dt}, J(\mathrm{PC})=23.0 \mathrm{~Hz}, J(\mathrm{PC})=5.4 \mathrm{~Hz}, \mathrm{C} 4), 159.3(\mathrm{~d}$, $J(\mathrm{PC})=11.1 \mathrm{~Hz}, \mathrm{C} 5), 139.4(\mathrm{~d}, J(\mathrm{PC})=23.3 \mathrm{~Hz} \mathrm{C} 3)$, $119.3(\mathrm{~d}, J(\mathrm{PC})=87.4 \mathrm{~Hz} \mathrm{C} 2), 154.7 \sim 123.1(\mathrm{Ph}$ and C6-C11), $73.6(\mathrm{~d}, J(\mathrm{PC})=117.4 \mathrm{~Hz}, \mathrm{C} 12)$; IR $v$ : 2011 (CO) $\mathrm{cm}^{-1}$; HRMS-ESI $(\mathrm{m} / \mathrm{z})$ calcd for $\left[\mathrm{C}_{93} \mathrm{H}_{71} \mathrm{IrOP}_{4}\right]^{2+}$ 760.7054 , found 760.7060 .

\subsection{3 铱杂碳龙配合物 6 的合成}

在 $50 \mathrm{~mL}$ 的反应瓶中加入铱碳龙配合物 4(500 mg, $0.31 \mathrm{mmol}) 、$ 二水合氧化三甲胺 $(69 \mathrm{mg}, 0.62 \mathrm{mmol}$ )和四 丁基氯化铵 $(86 \mathrm{mg}, 0.31 \mathrm{mmol})$, 用 $15 \mathrm{~mL}$ 二氯甲烷溶 解, 在常温下搅拌 $20 \mathrm{~min}$. 将反应液浓缩、乙醚洗涤 $(3 \times 20 \mathrm{~mL})$ 和真空干燥后得到 $429 \mathrm{mg}$ 绿色固体 6 , 产率 为 $90 \% .{ }^{1} \mathrm{H} \mathrm{NMR}\left(600.1 \mathrm{MHz}, \mathrm{CD}_{2} \mathrm{Cl}_{2}\right) \delta: 12.94(\mathrm{~s}, 1 \mathrm{H}$, $\mathrm{C} 14 H), 11.23(\mathrm{~d}, J(\mathrm{PH})=22.9 \mathrm{~Hz}, 1 \mathrm{H}, \mathrm{C} 1 H), 8.33$ (br, $1 \mathrm{H}$, $\mathrm{C} 3 H), 6.38 \sim 7.32(64 \mathrm{H}, \mathrm{Ph}) ;{ }^{31} \mathrm{P}\left\{{ }^{1} \mathrm{H}\right\}$ NMR $(242.9 \mathrm{MHz}$, $\left.\mathrm{CD}_{2} \mathrm{Cl}_{2}\right) \delta: 9.08\left(\mathrm{~s}, \mathrm{CPPh}_{3}\right), 6.89\left(\mathrm{~s}, \mathrm{CPPh}_{3}\right),-3.67(\mathrm{~s}$, $\left.\operatorname{Ir} P \mathrm{Ph}_{3}\right) ;{ }^{13} \mathrm{C}\left\{{ }^{1} \mathrm{H}\right\}$ NMR (150.9 MHz, $\mathrm{CD}_{2} \mathrm{Cl}_{2}$, plus ${ }^{13} \mathrm{C}$-dept 135, ${ }^{1} \mathrm{H}-{ }^{13} \mathrm{C}$ HSQC and ${ }^{1} \mathrm{H}_{-}{ }^{13} \mathrm{C}$ HMBC) $\delta$ : 225.1 (br, C14), 197.3 (br, C1), 175.4 (d, $J(\mathrm{PC})=14.9 \mathrm{~Hz}, \mathrm{C} 13), 163.2$ (dt, $J(\mathrm{PC})=20.5 \mathrm{~Hz}, J(\mathrm{PC})=3.4 \mathrm{~Hz}, \mathrm{C} 4), 154.4(\mathrm{~d}, J(\mathrm{PC})=$ $12.9 \mathrm{~Hz}, \mathrm{C} 5), 149.8 \sim 120.3$ (C3, C6-C11 and $\mathrm{Ph}), 133.2$ $(\mathrm{d}, J(\mathrm{PC})=26.3 \mathrm{~Hz}, \mathrm{C} 3), 121.7(\mathrm{~d}, J(\mathrm{PC})=86.1 \mathrm{~Hz}, \mathrm{C} 2)$, $66.4(\mathrm{~d}, J(\mathrm{PC})=119.8 \mathrm{~Hz} \mathrm{C} 12)$; HRMS-ESI $(\mathrm{m} / \mathrm{z})$ calcd for $\left[\mathrm{C}_{88} \mathrm{H}_{67} \mathrm{IrClP}_{4}\right]^{+}$1451.3506, found 1451.3552.

\subsection{4 铱杂碳龙配合物 7 的合成}

在 $50 \mathrm{~mL}$ 的反应瓶中加入铱碳龙配合物 $\mathbf{5}(304 \mathrm{mg}$, $0.18 \mathrm{mmol}$ 、二三水合氧化三甲胺 $(40 \mathrm{mg}, 0.36 \mathrm{mmol}$ )和四 丁基氯化铵 $(50 \mathrm{mg}, 0.18 \mathrm{mmol})$, 用 $10 \mathrm{~mL}$ 二氯甲烷溶 
解, 在常温下搅拌 $9 \mathrm{~h}$. 将反应液浓缩、乙醚洗涤 $(3 \times 20$ $\mathrm{mL}$ ) 和真空干燥后得到 $263 \mathrm{mg}$ 绿色固体 7, 产率为 $91 \%$. ${ }^{1} \mathrm{H}$ NMR (600.1 MHz, $\left.\mathrm{CD}_{2} \mathrm{Cl}_{2}\right) \delta: 10.94(\mathrm{~d}, J(\mathrm{PH})=21.1$ $\mathrm{Hz}, 1 \mathrm{H}, \mathrm{C} 1 H), 8.32$ (br, 1H, C3H), $7.82 \sim 6.17$ (69H, Ph); ${ }^{31} \mathrm{P}\left\{{ }^{1} \mathrm{H}\right\}$ NMR $\left(242.9 \mathrm{MHz}, \mathrm{CD}_{2} \mathrm{Cl}_{2}\right) \delta: 9.08\left(\mathrm{~s}, \mathrm{CPPh}_{3}\right)$, $7.47\left(\mathrm{~s}, \mathrm{CPPh}_{3}\right),-9.76$ (br, $\left.\operatorname{Ir} P \mathrm{Ph}_{3}\right) ;{ }^{13} \mathrm{C}\left\{{ }^{1} \mathrm{H}\right\} \quad \mathrm{NMR}$ (150.9 MHz, $\mathrm{CD}_{2} \mathrm{Cl}_{2}$, plus ${ }^{13} \mathrm{C}$-dept $135,{ }^{1} \mathrm{H}^{-13} \mathrm{C}$ HSQC and ${ }^{1} \mathrm{H}-{ }^{13} \mathrm{C}$ HMBC) $\delta: 233.2$ (br, C14), 196.1 (br, C1), 173.1 $(\mathrm{d}, J(\mathrm{PC})=11.4 \mathrm{~Hz}, \mathrm{C} 13), 159.9(\mathrm{dt}, J(\mathrm{PC})=23.9 \mathrm{~Hz}$, $J(\mathrm{PC})=5.6 \mathrm{~Hz}, \mathrm{C} 4), 155.3(\mathrm{~d}, J(\mathrm{PC})=12.8 \mathrm{~Hz}, \mathrm{C} 5), 150.4$ $(\mathrm{d}, J(\mathrm{PC})=12.6 \mathrm{~Hz}, \mathrm{C} 11), 134.6 \sim 119.7(\mathrm{C} 6-\mathrm{C} 10$ and $\mathrm{C} 3$ and $\mathrm{C} 2$ and $\mathrm{Ph}), 132.3(\mathrm{~d}, J(\mathrm{PC})=25.7 \mathrm{~Hz}, \mathrm{C} 3), 121.3(\mathrm{~d}$, $J(\mathrm{PC})=86.5 \mathrm{~Hz} \mathrm{C} 2), 66.1(\mathrm{~d}, J(\mathrm{PC})=119.1 \mathrm{~Hz} \mathrm{C} 12)$; HRMS-ESI $(\mathrm{m} / \mathrm{z})$ calcd for $\left[\mathrm{C}_{92} \mathrm{H}_{71} \mathrm{IrClP}_{4}\right]^{+} 1527.3819$, found 1527.3899 .

\subsection{5 铱杂碳龙配合物 8 的合成}

在 $50 \mathrm{~mL}$ 的反应瓶中加入铱碳龙配合物 $4(500 \mathrm{mg}$, $0.31 \mathrm{mmol}) 、$ 二水合氧化三甲胺 $(69 \mathrm{mg}, 0.62 \mathrm{mmol})$, 用 $15 \mathrm{~mL}$ 二氯甲烷溶解, 随后加入环己基异腈 $(77 \mu \mathrm{L}, 0.62$ $\mathrm{mmol}$ ), 在常温下摚拌 $2 \mathrm{~h}$. 将反应液浓缩、乙醚洗涤 $(3 \times 20 \mathrm{~mL})$ 和真空干燥后得到 $446 \mathrm{mg}$ 绿色固体 8 , 产率 为 $85 \% .{ }^{1} \mathrm{H} \mathrm{NMR}\left(500.1 \mathrm{MHz}, \mathrm{CD}_{2} \mathrm{Cl}_{2}\right) \delta: 11.25(\mathrm{~s}, 1 \mathrm{H}$, $\mathrm{C} 14 H), 10.04(\mathrm{~d}, J(\mathrm{PH})=21.9 \mathrm{~Hz}, 1 \mathrm{H}, \mathrm{C} 1 H), 8.57(\mathrm{~s}, 1 \mathrm{H}$, $\mathrm{C} 3 H), 7.94 \sim 6.35(64 \mathrm{H}, \mathrm{Ph}), 3.42 \sim 3.38(\mathrm{~m}, 1 \mathrm{H}, \mathrm{C} 16 H)$, $0.76 \sim 1.37(10 \mathrm{H}, \mathrm{CyH}) ;{ }^{31} \mathrm{P}\left\{{ }^{1} \mathrm{H}\right\} \mathrm{NMR}(202.5 \mathrm{MHz}$, $\left.\mathrm{CD}_{2} \mathrm{Cl}_{2}\right): \delta 11.80\left(\mathrm{~s}, \mathrm{CPPh}_{3}\right), 6.41\left(\mathrm{~s}, \mathrm{C} P \mathrm{Ph}_{3}\right), 2.00(\mathrm{~s}$, $\left.\mathrm{Ir} P P h_{3}\right) ;{ }^{13} \mathrm{C}\left\{{ }^{1} \mathrm{H}\right\}$ NMR $\left(125.8 \mathrm{MHz}, \mathrm{CD}_{2} \mathrm{Cl}_{2}\right.$, plus ${ }^{13} \mathrm{C}$-dept 135, ${ }^{1} \mathrm{H}-{ }^{13} \mathrm{C}$ HSQC and ${ }^{1} \mathrm{H}-{ }^{13} \mathrm{C}$ HMBC) $\delta: 204.3$ (br, C14), 182.6 (br, C1), 175.2 (d, $J(\mathrm{PC})=13.3 \mathrm{~Hz}, \mathrm{C} 13), 171.3$ (td, $J(\mathrm{PC})=24.5 \mathrm{~Hz}, J(\mathrm{PC})=3.6 \mathrm{~Hz} \mathrm{C} 4), 157.9(\mathrm{~d}, J(\mathrm{PC})=$ $11.5 \mathrm{~Hz}, \mathrm{C} 5), 152.9$ (d, $J(\mathrm{PC})=12.2 \mathrm{~Hz}, \mathrm{C} 15), 138.0$ (d, $J(\mathrm{PC})=25.2 \mathrm{~Hz} \mathrm{C} 3), 135.1 \sim 122.2(\mathrm{Ph}, \mathrm{C} 6-\mathrm{C} 11), 120.5$ $(\mathrm{d}, J(\mathrm{PC})=87.4 \mathrm{~Hz}, \mathrm{C} 2), 70.7(\mathrm{~d}, J(\mathrm{PC})=119.0 \mathrm{~Hz} \mathrm{C} 12)$, 55.40 (s, C16), 31.91 23.19 (CyC); IR v: $2169(\mathrm{CN})$ $\mathrm{cm}^{-1}$; HRMS-ESI $(\mathrm{m} / \mathrm{z})$ calcd for $\left[\mathrm{C}_{93} \mathrm{H}_{78} \mathrm{IrNP}_{4}\right]^{2+}$ 763.2368, found 763.2388.

\subsection{6 铱杂碳龙配合物 9 的合成}

在 $50 \mathrm{~mL}$ 的反应瓶中加入铱碳龙配合物 $\mathbf{4}(500 \mathrm{mg}$, $0.31 \mathrm{mmol}) 、$ 二水合氧化三甲胺 $(69 \mathrm{mg}, 0.62 \mathrm{mmol})$, 用 $15 \mathrm{~mL}$ 二氯甲烷溶解, 随后加入苯乙炔 $(170 \mu \mathrm{L}, 1.55$ $\mathrm{mmol}$ ), 在常温下摚拌 $2 \mathrm{~h}$. 将反应液浓缩、乙醚洗涤 $(3 \times 20 \mathrm{~mL})$ 和真空干燥后得到 $397 \mathrm{mg}$ 绿色固体 9, 产率 为 $80 \% .{ }^{1} \mathrm{H} \mathrm{NMR}\left(500.1 \mathrm{MHz}, \mathrm{CDCl}_{3}\right) \delta: 12.34(\mathrm{~s}, 1 \mathrm{H}$, $\mathrm{C} 14 H), 10.60(\mathrm{~d}, J(\mathrm{PH})=23.0 \mathrm{~Hz}, 1 \mathrm{H}, \mathrm{C} 1 H), 8.33(\mathrm{br}, 1 \mathrm{H}$, $\mathrm{C} 3 H), 7.81 \sim 6.18(69 \mathrm{H}, \mathrm{Ph}) ;{ }^{31} \mathrm{P}\{1 \mathrm{H}\} \mathrm{NMR}(202.5 \mathrm{MHz}$, $\left.\mathrm{CDCl}_{3}\right) \delta: 9.98(\mathrm{~s}, \mathrm{CPPh}), 6.24(\mathrm{~s}, \mathrm{CPPh}),-0.89$ (s, $\left.\operatorname{Ir} P \mathrm{Ph}_{3}\right) ;{ }^{13} \mathrm{C}\left\{{ }^{1} \mathrm{H}\right\}$ NMR $\left(125.8 \mathrm{MHz}, \mathrm{CDCl}_{3}\right.$, plus ${ }^{13} \mathrm{C}$-dept $135,{ }^{1} \mathrm{H}_{-}{ }^{13} \mathrm{C}$ HSQC and ${ }^{1} \mathrm{H}_{-}{ }^{13} \mathrm{C}$ HMBC) $\delta: 218.7$ (br, C14),
189.9 (br, C1), 181.1 (d, J(PC)=28.2 Hz, C13), 177.7 (d, $J(\mathrm{PC})=14.6 \mathrm{~Hz}, \mathrm{C} 5), 157.4(\mathrm{dt}$, apparant $\mathrm{d}, J(\mathrm{PC})=13.3$ $\mathrm{Hz}, \mathrm{C} 4), 150.3 \sim 119.9$ (C6-C11 and $\mathrm{Ph}), 135.4$ (d, J(PC) $=26.5 \mathrm{~Hz}, \mathrm{C} 3), 124.3(\mathrm{~d}, J(\mathrm{PC})=89.8 \mathrm{~Hz} \mathrm{C} 2), 116.5(\mathrm{~s}$, $\mathrm{C} 16), 99.1(\mathrm{t}, J(\mathrm{PC})=17.0 \mathrm{~Hz} \mathrm{C} 15), 65.1(\mathrm{~d}, J(\mathrm{PC})=120.9$ $\mathrm{Hz}$ C12); HRMS-ESI $(\mathrm{m} / \mathrm{z})$ calcd for $\left[\mathrm{C}_{94} \mathrm{H}_{72} \mathrm{IrP}_{4}\right]^{+}$ 1518.4242 , found, 1518.4249 .

\section{References}

[1] Selected reviews: (a) He, G.; Xia, H.; Jia, G. Chin. Sci. Bull. 2004 49, 1543. (b) Landorf, C. W.; Haley, M. M. Angew. Chem., Int. Ed. 2006, 45, 3914. (c) Chen, J.; Jia, G. Coord. Chem. Rev. 2013, 257, 2491. (d) Cao, X.-Y.; Zhao, Q.; Lin, Z.; Xia, H. Acc. Chem. Res. 2014, 47, 341. (e) Frogley, B. J.; Wright, L. J. Coord. Chem. Rev. 2014, 270-271, 151. (f) Fernández, I.; Frenking, Gernot.; Merino, G. Chem. Soc. Rev. 2015, 44, 6452. (g) Frogley, B. J.; Wright, L. J. Chem. -Eur. J. 2018, 24, 2025. (h) Hua, Y.; Zhang, H.; Xia, H. Chin. J. Org. Chem. 2018, 38, 11 (in Chinese). (华显辉, 张弘, 夏海平, 有机化学, 2018, 38, 11)

[2] Selected reviews: (a) Jia, G. Acc. Chem. Res. 2004, 37, 479. (b) Chen, J.; He, G.; Jia, G. Chin. J. Org. Chem. 2013, 33, 792 (in Chinese). (陈江溪, 何国梅, 贾国成, 有机化学, 2013, 33, 792) (c) Jia, G. Organometallics 2013, 32, 6852.

[3] (a) Paneque, M.; Posadas, C. M.; Poveda, M. L.; Rendón, N.; Salazar, V.; Oñate, E.; Mereiter, K. J. Am. Chem. Soc. 2003, 125, 9898 (b) Paneque, M.; Posadas, C. M.; Poveda, M. L.; Rendón, N.; Santos, L. L.; Álvarez, E.; Salazar, V.; Mereiter, K.; Oñate, E. Organometallics 2007, 26, 2403. (c) Vivancos, Á.; Hernández, Y. A. Paneque, M.; Poveda, M. L.; Salazar, V.; Álvarez, E. Organometallics 2015, 34, 177.

[4] (a) He, G.; Chen, J.; Xia, H.; Sci. Bull. 2016, 61, 430. (b) Zhou, X.; Zhang, H. Chem. Eur. J. 2018, 24, 8962. (c) Wang, H.; Zhou, X.; Xia, H. Chin. J. Chem. 2018, 36, 93.

[5] Wei, J.; Zhang, W.; Xi, Z. Chem. Sci. 2018, 9, 560.

[6] (a) Zhang, Y.; Wei, J.; Chi, Y.; Zhang, X.; Zhang, W.-X.; Xi, Z. J. Am. Chem. Soc. 2017, 139, 5309. (b) Liu, L.; Zhu, M.; Yu, H.-T.; Zhang, W.-X.; Xi, Z. J. Am. Chem. Soc. 2017, 139, 13688. (c) Zhang, Y.; Wei, J.; Zhu, M.; Chi, Y.; Zhang, W.-X.; Ye, S.; Xi, Z. Angew. Chem., Int. Ed. 2019, 58, 9625.

[7] (a) Wei, J.; Zhang, Y.; Chi, Y.; Liu, L.; Zhang, W.-X.; Xi, Z. J. Am. Chem. Soc. 2016, 138, 60. (b) An, K.; Shen, T.; Zhu, J. Organometallics 2017, 36, 3199. (c) Huang, Z.; Zhang, Y.; Zhang, W.-X.; Xi, Z. Organometallics 2019, 38, 2807.

[8] (a) Lv, Z.-J.; Huang, Z.; Shen, J.; Zhang, W.-X.; Xi, Z. J. Am. Chem. Soc. 2019, 141, 20547. (b) Wang, K.; Zhou, X. Chin. J. Org. Chem. $\mathbf{2 0 2 0}, 40,1084$ (in Chinese). (主凯, 周锡庚, 有机化学, 2020, 40, 1084)

[9] (a) Frogley, B. J.; Wright, L. Angew. Chem., Int. Ed. 2017, 56, 143. (b) Ruan, W.; Leung, T.-F.; Shi, C.; Lee, K. H.; Sung, H. H. Y.; Williams, I. D.; Lin, Z.; Jia, G. Chem. Sci. 2018, 9, 5944. (c) Talavera, M. Peña-Gallego, A. Alonso-Gómez, J. L. Bolaño, S.; Chem. Commun., 2018, 54, 10974. (d) Zhang, M.-X.; Xu, Z.; Lu, T.; Yin, J.; Liu, S. H. Chem. Eur. J. 2018, 24, 14891. (e) Zhang, M.-X.; Zhang, J.; Jin, X.; Sun, X.; Yin, J.; Hartl, F.; Liu, S. H. Chem. Eur. J. 2018, 24, 18998. (f) Hu, Y. X.; Zhang, J.; Wang, X.; Lu, Z.; Zhang, F.; Yang, X.; Ma, Z.; Yin, J.; Xia, H.; Liu, S. H. Chem. Sci. 2019, 10, 10894. (g) Chu, Z.; He, G.; Cheng, X.; Deng, Z.; Chen, J. Angew. Chem., Int. Ed. 2019, 58, 9174.

[10] (a) Zhu, C.; Xia, H. Acc. Chem. Res. 2018, 51, 1691. (b) Luo, M.; Hua, Y.; Zhuo, K.; Long, L.; Lin, X.; Deng, Z.; Lin, Z.; Zhang, H.; Chen, D.; Xia, H. CCS Chem. 2020, 2, 758. (c) Lin, J.; Xu, Q.; Lin, X.; Hua, Y.; Chen, D.; Ruan, Y.; Zhang, H.; Xia, H. Chin. J. Chem. $\mathbf{2 0 2 0}, 38,1273$.

[11] Zhu, C.; Li, S.; Luo, M.; Zhou, X.; Niu, Y.; Lin, M.; Zhu, J.; Cao, Z.; Lu, X.; Wen, T.; Xie, Z.; Schleyer, P. v. R.; Xia, H. Nat. Chem. 2013, 5,698 .

[12] (a) Zhu, C.; Yang, Y.; Luo, M.; Yang, C.; Wu, J.; Chen, L.; Liu, G.; Wen, T.; Zhu, J.; Xia, H. Angew. Chem., Int. Ed. 2015, 54, 6181. (b) Yang, C.; Lin, G.; Zhu, C.; Pang, X.; Wang, X.; Li, X.; Wang, B.; Xia, H.; Liu, G. J. Mater. Chem. B, 2018, 6, 2528. (c) Zhou, X.; 
Pang, X.; Nie, L.; Zhu, C.; Zhuo, K.; Zhuo, Q.; Chen, Z.; Liu, G.; Zhang, H.; Lin, Z.; Xia, H. Nat. Commun. 2019, 10, 1488.

[13] Zhu, C.; Yang, C.; Wang, Y.; Lin, G.; Yang, Y.; Wang, X.; Zhu, J.; Chen, X.; Lu, X.; Liu, G.; Xia, H. Sci. Adv. 2016, 6, e1601031/1.

[14] Li, R.; Lu, Z.; Cai, Y.; Jiang, F.; Tang, C.; Chen, Z.; Zheng, J.; Pi, J.; Zhang, R.; Liu, J.; Chen, Z.-B.; Yang, Y.; Shi, J.; Hong, W.; Xia, H. J. Am. Chem. Soc. 2017, 139, 14344.

[15] (a) Zhang, H.; Zhao, H.; Zhuo, K.; Hua, Y.; Chen, J.; He, X.; Weng, W.; Xia, H. Polym. Chem. 2019, 10, 386. (b) Chen, Y.; Yang, L.; Zheng, W.; Ouyang, P.; Zhang, H.; Ruan, Y.; Weng, W.; He, X.; Xia, H. ACS Macro Lett. 2020, 9, 344.

[16] Zhuo, Q.; Lin, J.; Hua, Y.; Zhou, X.; Shao, Y.; Chen, S.; Chen, Z.; Zhu, J.; Zhang, H.; Xia, H. Nat. Commun. 2017, 8, 1912.

[17] (a) Zhuo, Q.; Zhang, H.; Hua, Y.; Kang, H.; Zhou, X.; Lin, X.; Chen, Z.; Lin, J.; Zhuo, K.; Xia, H. Sci. Adv. 2018, 4, eaat0336. (b) Li, J.; Kang, H.; Zhuo, K.; Zhuo, Q.; Zhang, H.; Lin, Y.-M.; Xia, H. Chin. J. Chem. 2018, 36, 1156 .

[18] Zhuo, Q.; Zhang, H.; Ding, L.; Lin, J.; Zhou, X.; Hua, Y.; Zhu, J.; Xia, H. iScience 2019, 19, 1214.

[19] (a) Schröder, F. G.; Sundermeyer, J. Organometallics 2015, 34, 1017. (b) Hariharan, P. S.; Mariyatra, M. B.; Mothi, E. M.; Neels, A. Rosair, G.; Anthony, S. P. New. J. Chem. 2017, 41, 4592.

[20] (a) Bleeke, J. R.; Behm, R. J. Am. Chem. Soc. 1997, 119, 8503. (b) Clark, G. R.; Johns, P. M.; Roper, W. R.; Wright, L. J. Organometal- lics 2008, 27, 451.

[21] Wu, H.-P.; Ess, D. H.; Lanza, S.; Weakley, T. J. R.; Houk, K. N.; Baldridge, K. K.; Haley, M. M. Organometallics 2007, 26, 3957.

[22] (a) O'Connor, J. M.; Pu, L. J. Am. Chem. Soc. 1990, 112, 9663. (b) O'Connor, J. M.; Pu, L.; Chadha, R. Angew. Chem., Int. Ed. Engl. 1990, 29, 543.

[23] Zhu, C.; Luo, M.; Zhu, Q.; Zhu, J.; Schleyer, P. v R.; Wu, J. I.-C.; Lu, X.; Xia, H. Nat. Commun. 2014, 5, 3265.

[24] (a) Ilg, K.; Werner, H. Organometallics 2001, 20, 3782. (b) Chin, C. S.; Kim, M.; Lee, H.; Noh, S.; Ok, K. M. Organometallics 2002, 21, 4785. (c) Torres, O.; Martín, M.; Sola, E. Organometallics 2010, 29, 3201.

[25] (a) Li, J.; Lin, Y.-M.; Zhang, H.; Chen, Y.; Lin, Z.; Xia, H. Chem. -Eur. J. 2019, 25, 5077. (b) Wu, F.; Huang, W.; Zhuo, K.; Hua, Y.; Lin, J.; He, G.; Chen, J.; Nie, L.; Xia, H. Chin. J. Org. Chem. 2019 39, 1743 (in Chinese). (吴凡, 黄文超, 卓凯玥, 华显晖, 林剑锋, 何国梅, 陈江溪, 聂立铭, 夏海平, 有机化学, 2019, 39, 1743) (c) Lu, Z.; Zhu, Q.; Cai, Y.; Chen, Z.; Zhuo, K.; Zhu, J.; Zhang, H.; Xia, H. Sci. Adv. 2020, 6, eaay2535.

[26] Bedard, T. C.; Moore, J. S. J. Am. Chem. Soc. 1995, 117, 10662.

[27] Dubé, P.; Toste, F. D. J. Am. Chem. Soc. 2006, 128, 12062.

[28] Kaiser, R. P.; Hessler, F.; Mosinger, J.; Císařová, I.; Kotora, M. Chem. Eur. J. 2015, 21, 13577.

(Yang, X.) 